\title{
Studying the populations of our Galaxy using the kinematics of sdB stars ${ }^{\star}$
}

\author{
M. Altmann ${ }^{1,2}$, H. Edelmann ${ }^{2}$, and K. S. de Boer ${ }^{1}$ \\ 1 Sternwarte der Universität Bonn, Auf dem Hügel 71, 53121 Bonn, Germany \\ e-mail: deboer@astro.uni-bonn.de \\ 2 Dr. Remeis-Sternwarte, Sternwartstr. 7, 96049 Bamberg, Germany \\ e-mail: edelmann@sternwarte.uni-erlangen.de
}

Received 20 May 2003 / Accepted 10 October 2003

\begin{abstract}
We have analysed the kinematics of a sample of 114 hot subdwarf stars. For 2/3 of the stars, new proper motions, spectroscopic and photometric data are presented. The vast majority of the stars show a kinematic behaviour that is similar to that of Thick Disk stars. Some stars have velocities rather fitting to solar, i.e. Thin Disk, kinematics. About $\sim 15$ objects have orbital velocities which differ considerably from those of Disk stars. These are members of the Galactic Halo. We investigated the velocity dispersions and calculated the orbits. Most stars feature orbits with disk character (eccentricity of less than 0.5), a few reach far above the Galactic plane and have very eccentric orbits (eccentricity of more than 0.7 ). The intermediate eccentricity range is poorly populated. This seems to indicate that the (Thick) Disk and the Halo are kinematically disjunct. Plotting a histogram of the orbit data points along $z$ leads to the $z$-distance probability distribution of the star; doing this for the whole sample leads to the $z$-distance probability distribution of the sample. The logarithmic histogram shows two slopes, each representing the scale height of a population. The disk component has a scale height of $0.9( \pm 0.1) \mathrm{kpc}$, which is consistent with earlier results and is similar to that of the Thick Disk. The other slope represents a component with a scale height $\sim 7 \mathrm{kpc}$, a much flatter gradient than for the disk component. This shows that the vast majority of the sdBs are disk stars, but a Halo minority is present, too. The kinematic history and population membership of the sdB stars on the whole is different from that of the cooler HBA stars, which are predominantly or even exclusively Halo objects. This leads to the question, whether the Halo sdB stars are of similar origin as the HBA stars, or whether their kinematical behaviour possibly represents another origin, such as infalling stellar aggregates or inner disk events.
\end{abstract}

Key words. astrometry - stars: kinematics - stars: horizontal branch - stars: population II - Galaxy: halo - Galaxy: structure

\section{Introduction}

To understand the structure and evolution of galaxies it is essential to study the gaseous and stellar components of the Milky Way. No other large galaxy gives us access to the spatial distribution and kinematics of stars in such detail. A vital aspect is the study of older stars, because it brings insight into the formation and evolution of the Galaxy and thus of galaxies in general (assuming that our Galaxy is typical with no or only small peculiarities).

One approach to studying the distribution of stars is to conduct a star count over all stars in fields at different Galactic latitudes such as done by Reid \& Majewski (1993). Fitting models to account for luminosity class, metallicity, completeness,

Send offprint requests to: M. Altmann, e-mail: maltmann@astro.uni-bonn.de

* Based on data obtained at the $1.54 \mathrm{~m}$ telescope of the European Southern Observatory, runs 58.D-0478, 60.D-0306, 62.L-0220，64.L-0077，64.H-0023(A)，65.H-0250，65.H-0341(A) and 68.D-0192(A). distributions and number densities of populations etc. to the raw results then leads to scale heights and space densities. This method relies on a very large number of stars, which means that the statistical errors are small. On the downside, these studies heavily rely on models, introducing uncertainties caused by possibly poorly known input parameters.

Therefore many studies use a certain well defined star type as a tracer rather than all available stars. In most cases these tracers are evolved stars, which, while being relatively rare, are bright so that studies using them extend deeper into the galaxy. Widely used objects are giants and horizontal branch (HB) stars (especially RR-Lyraes, because they are, as variables very easily identified). Nowadays the recent deep surveys enable studies using very low mass main sequence stars as tracers as well (Phleps et al. 2000).

Studies of the spatial distribution of a sample of stars give insight into the general structure of the Galaxy, e.g. revealing various populations of stars. Adding kinematical data gives us access to the motions of the stars forming these groups. Stars belonging to different populations show widely differing 
kinematical behaviour. Some components of the Milky Way are rapidly rotating with little dispersion in the velocities of the members while others show only little rotation but high dispersions. These differences between the populations give us evidence of how these parts of the Galaxy are formed. Such studies have been conducted for quite a variety of different object types, such as high proper motion stars (Carney et al. 1996), local dwarfs (Schuster \& Allen 1997) or globular clusters (Dinescu et al. 1997, 1999a,b). The kinematics of sdB stars have been studied by Colin et al. (1994), Thejll et al. (1997) and de Boer et al. (1997).

Blue subdwarf stars, such as sdB stars, are particularly suited for such a study because they have spectra that can be analysed with relatively simple methods. And since their spectra are quite unique, there are no other objects they can be easily confused with.

Horizontal Branch stars (HB) are core helium burning objects after the first red giant (RGB) phase. Their appearance depends on the mass of the hydrogen envelope they retain, while the He core mass is relatively constant over all types.

Extreme HB stars (EHB) such as sdB stars only have a very thin $\mathrm{H}$ envelope of less than $0.02 M_{\odot}$. HB stars hotter than about $10000 \mathrm{~K}$ have metal abundances heavily altered by effects of diffusion and levitation in their stable and nonconvective atmospheres, as has been found by many studies (Bonifacio et al. 1995; Moehler et al. 1999; Behr et al. 1999). Since the present element abundances do represent the initial metal content, information of the population membership of hot HB stars is only accessible through their spatial distribution and kinematics.

The study presented here is a continuation of the work done by de Boer et al. (1997), increasing the number of stars by a factor of almost three, adding some more local stars and stars from the HE-survey carried out by the Hamburger Sternwarte. These are on average a little further away than those of the PG-survey (Green et al. 1986) used in the earlier work, but on the whole at significantly larger $z$-heights as most of them are located at higher Galactic latitudes than the PG-stars used in de Boer et al. (1997). With this enlarged sample, one can therefore expect the probability to include Halo objects in the sample to be larger, because their relative density is higher than near the Sun where disk stars dominate.

Section 2 deals with the composition of our sample, data acquisition and reduction. In Sect. 2.1.1 we discuss possibly selection effects induced by the sample composition. The analysis of the kinematics and orbits is described in Sect. 3, the determination of a scale height from the orbits in Sect. 4. In Sect. 5 we discuss our results in a larger context. Finally Sect. 6 gives our conclusions and an outlook towards the future.

\section{Data and data reduction}

\subsection{The sample}

The sample of $114 \mathrm{sdB} / \mathrm{OB}$ stars (sdOB stars are hotter versions of sdB stars with $T_{\text {eff }}$ of more than about $32000 \mathrm{~K}$ featuring some He lines in their spectra. Since they are - unlike the even hotter sdO stars - generally believed to be HB-like
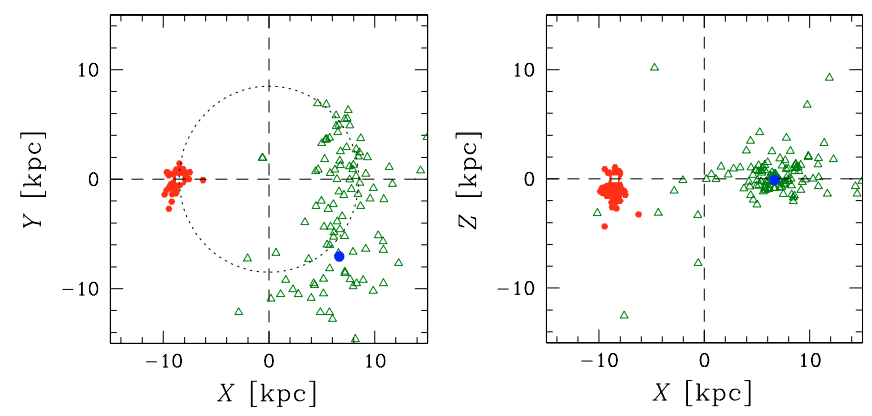

Fig. 1. Distribution of the stars of our sample today (full hexagons) and approximately half a revolution (100 Myr) earlier (open triangles) showing that our stars, now concentrated in a small volume, came in fact from all over the Galaxy. The left panel shows the distributions on the Galactic plane, the right panel shows the distributions perpendicular to the plane (along the $X$-axis). The full square and full circle show the position of the Sun today and $100 \mathrm{Myr}$ ago respectively. The circle on the left panel shows the present galactocentric distance of the Sun. The Galactic centre is in the middle of both diagrams, the dashed lines show the zero line for each coordinate.

stars we use the term sdB stars throughout this work) is composed of objects taken from several sources. 59 stars, located in the southern polar cap (SPC) of our galaxy have been taken from the Hamburg/ESO survey (HE). For these new data have been obtained. We further included the 41 objects published in de Boer et al. (1997), which were mainly taken from the Palomar Green catalogue (Green et al. 1986). 17 stars with Hipparcos or Tycho 2 data $^{1}$ have also been included as well as one star (PG 1716+426) whose kinematics was analysed by Geffert (1998).

\subsubsection{Selection effects due to sample composition?}

The sample of sdB stars discussed in this paper is the collection of all relatively nearby stars we could lay our hands on. The sole criterion was, that we should have or could obtain, for each sdB star its distance, its radial velocity and its proper motion. We have not aimed at obtaining and working with an observationally unbiased sample. To show that this will not cause any problems in the results is the goal of the discussion of this section. For the present positions of the stars of the sample see Fig. 1.

The main sources of our objects were the catalogues from the surveys PG and HE. The PG and HE surveys were conducted at high Galactic latitudes. These surveys are incomplete at the bright end (the PG for stars brighter than $B \sim 12 \mathrm{mag}$, the $\mathrm{HE} \sim 13 \mathrm{mag})^{2}$.

Thus nearby stars at high Galactic latitudes may be underrepresented. However, since stars presently nearby may venture

\footnotetext{
1 Three objects (HD 205805, CD -38 222, PG 1519+640) are in common with de Boer et al. (1997). We use here the Hipparcos or Tycho2 proper motion data which was not yet available at the time of publication of de Boer et al. (1997).

2 These upper limits are not explicitly stated in Green et al. (1986) but for the Hamburg survey they are stated in the description of the catalogues: http://www.hs.uni-hamburg.de/ EN/For/Eng/Sur/index.html
} 
in time to almost any Galactic location (see Fig. 1), our sample must contain sufficient presently distant stars which at other times would have been near the Sun being then that bright, that they would have escaped the PG and HE surveys.

The stars we found in the HIPPARcos data base, which are mainly from the SB-survey of blue SGP objects (Slettebak \& Brundage 1971), are relatively bright and thus nearby.

The catalogues available normally do not (or rarely) contain stars presently at low Galactic latitudes. All of those low $b$ stars having Thick Disk or Halo kinematics would at other times have been detected in surveys like PG and HE (see Fig. 1). Stars with that kind of kinematics are thus not underrepresented in our sample. Most of the stars presently in the disk (thus missing from our sample) and which do have disk kinematics would always have been missed. Thus stars with Thin Disk like kinematics are probably somewhat underrepresented in our sample.

Summarising, in spite of having used data from various special catalogues dealing with particular observational selections of all stars available, our sample is only lacking (to an unknown amount) in stars with Thin Disk kinematics.

\subsection{Obtaining the data}

The data has been acquired over the past few years, mainly from the ESO La Silla. For the 41 stars of de Boer et al. (1997) we took all of their data, except for a few cases. A few proper motions have been taken from the HIPPARCos catalogue (ESA 1997), some of the other data from various sources in the literature (see Sect. 2.3).

\subsubsection{Spectroscopic data}

The spectra of the southern HE-stars have been taken at La Silla, Chile, with the ESO 1.54 m Danish telescope using the Danish Faint Object Spectrograph and Camera (DFOSC) covering a spectral range from 3500-5500 $\AA$ and with the ESO $1.52 \mathrm{~m}$ telescope using the Boller and Chivens spectrograph covering a spectral range from 3500-7000 $\AA$. The spectral resolution is about 5.0 and $5.5 \AA$, respectively. In order to conduct the wavelength calibration, especially because radial velocities were to be derived, an emission lamp spectrum was taken after the object spectrum, while the telescope was in the same position as during the object exposure. For the flux calibration spectra of the Oke-standard star Feige 110 (Oke 1990) were obtained. Exposure times were between $120 \mathrm{~s}$ and $3600 \mathrm{~s}$, depending on the brightness of the object.

\subsubsection{Astrometric and photometric data}

For each object photometry in $B$ and $V$ is required. For the astrometry images in bands in the redder part of the spectrum are needed to minimise effects from differential refraction (see e.g. Brosche et al. 1989). Therefore images were taken in $B$, $V$ and $R$ passbands. In order to calibrate the proper motions to the extragalactic reference frame, deep and relatively wide field exposures of the fields surrounding the stars are also needed.
Data acquisition of CCD images for photometry and as second epoch material for the determination of proper motions has been combined wherever possible.

The data was obtained with the $1.54 \mathrm{~m}$ Danish telescope and the DFOSC focal reducer at La Silla in January 1999, October 1999 and September 2000. The exposure times were between $600 \mathrm{~s}$ and $900 \mathrm{~s}$ ( $V$ and $R$ only) for those obtained at La Silla. As the electronic gain of the CCD camera used for the La Silla images is such that the target star is overexposed in most cases, an additional short exposure was made with an exposure time of 5 to $120 \mathrm{~s}$ depending on the approximate magnitude of the star. To complete the photometry a short $B$ exposure was taken as well. On all nights Landolt standard stars (Landolt 1992) were taken at least twice per night, mostly three times per night.

\subsection{Data reduction and analysis}

\subsubsection{Spectroscopy: Radial velocities, $\log g$ and $T_{\text {eff }}$}

The spectra were reduced using the ESO-MIDAS package. After bias subtraction and flat field correction the background was subtracted and the one dimensional spectra were extracted from the two dimensional frames. Thereafter a wavelength calibration was performed with calibration spectra recorded immediately after each stellar spectrum. Then all wavelength calibrated spectra were corrected for atmospheric extinction using the extinction coefficients of La Silla (Tüg 1977).

The observed spectra (line profiles of Balmer, He I and He II lines) were then fitted to synthetic spectra calculated from fully line blanketed LTE model atmospheres (Heber et al. 2000) as well as hydrogen and helium blanketed NLTE model atmospheres (Napiwotzki 1997). The model spectra were convolved to match the spectrograph's resolution and shifted by the approximate radial velocity. The fitting was accomplished using the fitting routine of Lemke (1997), which is based on the procedure of Bergeron et al. (1992) and Saffer et al. (1994). The values of the fit parameters $T_{\text {eff }}, \log g$ and $n \mathrm{He}$, the helium abundance, will be published in Edelmann et al. (2003).

Spectroscopic distances were determined using the method of flux conservation. For this we first obtained the angular stellar diameter by comparing model atmospheric fluxes with the dereddened apparent $V$-magnitude. From the determined $\log g$, the radii of the stars are calculated. We assume the mass of the sdB stars to be $0.50 M_{\odot}$. The errors of $\log g$ are estimated to be $0.1 \mathrm{dex}$ and for $T_{\text {eff }} 2.5 \%$. The error in the photometry is about $0.02 \mathrm{mag}$. The error of the distances was determined by error propagation to be in the order of $10 \%$.

Finally radial velocities were derived from the centres of gravity for all Balmer and helium lines.

The radial velocity of each star was then calculated by averaging the obtained single line velocities. These then were transformed to heliocentric values. The resulting radial velocities have an error of about $30 \mathrm{~km} \mathrm{~s}^{-1}$.

Some of the stars show signs of companions in their spectra. These spectroscopically obvious secondary components contribute significantly to the continuum flux. The routine we employed to fit spectral models to the spectra critically 
depends on the local continuum. Therefore in the case of a binary star the continuum of the secondary star must be taken care of. As this is a rather tedious procedure and only a few stars are affected we did not include these in our final sample. This does not apply for those stars taken from the literature which were measured using other fitting techniques, or in the case of SB 744, where the secondary continuum was subtracted (Unglaub \& Bues 1990).

sdB stars may be the result of close binary evolution. This means that even many of those that do not show any sign of binarity in their spectrum may have a close companion. The companion star, being too faint to show up in the spectrum (through spectral lines or a redder continuum), must either be a white dwarf or a low mass main-sequence star. Such unseen companions do not play a role for the method of the determination of physical parameters. They however $d o$ play an important role for the radial velocity determination. Especially if the secondary star is a white dwarf of similar or even higher mass than that of the sdB primary, the measured radial velocity may have a large amplitude - in some cases over $200 \mathrm{~km} \mathrm{~s}^{-1}$. Indeed, recent studies have found many stars with variable radial velocities (see e.g. Maxted et al. 2001; Morales-Rueda et al. 2003 etc.). As far as possible we have used systemic radial velocities published in those studies and some by Marsh (priv. comm.). Unfortunately we only have access to a few values - for most of our stars we only have a single value. Luckily the amplitudes of the majority of the variable radial velocities are far less dramatic, in the order of $50 \mathrm{~km} \mathrm{~s}^{-1}$ or less. The percentage of close binaries is by no means certain - the most recent (Napiwotzki, Edelmann, priv. comm.) results show that only about $30 \%$ of the $\mathrm{sdB}$ stars do show a variation in their radial velocity indeed, in contrast to two thirds as estimated in earlier studies. At present we cannot quantify the influence of variable radial velocities on our results; we will have to bear this problem in mind, and try to determine the systemic radial velocities of objects with suspicious (i.e. very large) values in the future.

\subsubsection{Photometry}

The basic CCD data reduction, i.e. bias subtraction, flatfield correction $^{3}$ and correction of bad pixels and rows were accomplished using the IRAF data reduction package which was also used for the subsequent photometric analysis.

The photometric measurements were conducted using aperture photometry. The aperture diameter used was 14", which is the size also used by Landolt (1992) for his standard star photometry as used to calibrate our measurements. For those stars, for which we could not derive an accurate magnitude (because the night was not photometric) we used values from the

\footnotetext{
${ }^{3}$ Flat field correction was somewhat cumbersome. There were noticeably differing large scale structures on the twilight flats made in the evening and during the morning twilight, resulting in a residual gradient remaining on the flat field corrected images as well. We therefore combined the long exposed object frames such that all stars could be eliminated. This sum image has the true flat gradient. The twilight flat was blurred and the original twilight flat was divided by the blurred one to get the small scale structure. This was then added to the scienceframe flat, to be used for the final flat correction.
}

literature, and in some cases if literature values were not available, their bjdss-magnitudes.

The majority of our stars is located at intermediate to very high Galactic latitudes. Nevertheless the photometry must be extinction corrected, to minimise systematic distance effects. Since almost all stars of our sample are located at $|z|>200 \mathrm{pc}$, thus likely above the Galactic dust layer, one can assume that all interstellar extinction is in front of the star. Therefore we decided to use reddening maps, such as those from Schlegel et al. (1998) or Burstein \& Heiles (1982). The latter have no data for about $30 \%$ of our stars, namely those located near the SGP.

The values taken from Schlegel et al. (1998) are a little larger than those of Burstein \& Heiles (1982). Subtracting 0.02 mag from their values, which the authors suggest to make the data comparable seems to overdo it. As a good compromise we decided to take the Schlegel et al. (1998) reddenings, reduced by $0.01 \mathrm{mag}$. The very few resulting negative values were taken as $E_{B-V}=0$.

For the majority of the stars $E_{B-V}$ was below $0.02 \mathrm{mag}$, a few had an $E_{B-V}$ of between 0.04 and 0.05 . This means that a residual $E_{B-V}$ will cause an error in the distances which is small compared to the influences of the other errors. For the stars for which we took the data from the literature we also used the extinction values from there.

\subsubsection{Astrometry}

1st epoch material: The biggest problem encountered when determining proper motions of stars is the availability of suitable 1st epoch material. Before 1950 photographic plates taken are mostly only of areas of special interest with conspicuous objects. So one has to rely on whole sky surveys, such as the Palomar Observatory Sky Survey (POSS), and its southern extension, the UK-Schmidt Survey. The latter was completed in the 1970's and early 1980's while the POSS was accomplished between 1948 and 1958. In recent times several scanned versions of these plates became available, like the DSS or the APM (Irwin \& McMahon 1992; Irwin et al. 1994). The APM catalogue has been used for the determination of proper motions of objects north of the equator in the past (de Boer et al. 1997). Unfortunately for $\delta<-15^{\circ}$, the APM relies on recent plates, rendering it unsuitable as first epoch material. Therefore we took positions derived from the DSS. These scans have an image scale of $1.7^{\prime \prime} /$ pix and are made from the blue plates of the UK Schmidt-survey. This could present a problem, because blue light is more affected by differential refraction than red light. However, the plates were taken at very low airmass, most of them near the meridian. The declinations of our objects are between $-17^{\circ}$ and $-55^{\circ}$, so they are not more than $25^{\circ}$ off zenith when passing the meridian.

Of the position determining methods generally used to get the plate coordinates, DAOPHOT is presumably not the best for digitised photographic plates, because it uses a PSF, and the PSFs of all photographic stellar images are different, in contrast to those of CCD images. For this reason we decided to use SEXTRACTOR (Bertin \& Arnouts 1996). Comparing positions derived using DAOPHOT and SEXTRACTOR shows 
a good agreement for well exposed stars, with $\sigma(\Delta x, \Delta y) \simeq$ 0.02 pix, or $0.035^{\prime \prime}$. In order to compare DSS and APM positions, we derived proper motions for 9 stars taken from a field north of the equator using both sources of first epoch material. The standard deviations of the differences between the pairs of proper motions was $\sim 2.5 \mathrm{mas} / \mathrm{yr}$. So we conclude that the error of the proper motions of the southern stars, which have a much smaller epoch difference, is about 4-5 mas/yr. Adding the error of the galaxy calibration, which is in the order of 1-2 mas/yr, the proper motions derived from DSS first epoch material are good to about 5-6 mas/yr.

2nd epoch material: As second epoch material, the same CCD-exposures were used as for the photometry (see Sect. 2.2.2). The DFOSC data has an image scale of $0.39^{\prime \prime} /$ pix. For the determination of plate coordinates, SEXTRACTOR was used for the CCD-images as well.

Reference catalogues: The third item required for the derivation of proper motions is a reference catalogue of the field preferably including proper motions of its stars. However, for small-field astrometry, e.g. with CCD data, there are not enough stars with proper motions in a typical field. Even the Tycho- 2 catalogue provides only about 1 to 5 entries in a DFOSC field of $13.7^{\prime} \times 13.7^{\prime}$, almost all of which are saturated even on the shortest exposures. This means that we had to rely on catalogues without proper motions and had to accept that the resulting proper motions are relative ones, which have to be calibrated to an absolute reference frame. We accomplish this by using the background galaxies in the field which have by definition a zero proper motion (see below).

We created a reference catalogue based on our CCD-data using the USNO-A2.0 catalogue (Monet 1998) as start catalogue.

Reduction: The astrometric reduction was performed using the BAP-software of Michael Geffert (see e.g. Geffert et al. 1997). In a two step iteration we first determined celestial positions for each plate/CCD-frame, which were then averaged to give a catalogue with positions and proper motions. This catalogue was then in turn used as new reference catalogue for the second iteration. For the plate reduction we used a plate model with 3rd order terms.

The resulting errors (based alone on the second epoch, since there is only one first epoch position) are in the order of $1 \mathrm{mas} / \mathrm{yr}$ with only a few stars having an error larger than $2 \mathrm{mas} / \mathrm{yr}$.

Calibration: As indicated above, the resulting proper motions are relative only. Therefore we transfer them to an absolute reference frame using the background galaxies. For this one determines the apparent galaxy proper motion centroid and subtracts this from the stellar proper motions. The galaxies are identified and separated from stars by criteria of FWHM of their images, SEXTRACTORS stellarity index and additional user defined selections to account for the larger error of FWHM for faint objects. This method is well established and often used in galaxy searches. For each star we found between 15 and 120 galaxies, mostly between 40 and 70 . The limiting factor is the 1st epoch material; the long CCD exposures, which were used for galaxy searching, usually yielded several hundred galaxies.

Unfortunately most background galaxies are very faint, show too much intrinsic (spectral dependent) structure, and are generally undersampled in the digitised photographic plates. Therefore the derived proper motions scatter considerably. To get rid of outliers we expunged objects deviating by more than $2 \sigma$. This gave us in general a standard deviation of 10 to $15 \mathrm{mas} / \mathrm{yr}$ and a resulting error of 0.5 to $2.5 \mathrm{mas} / \mathrm{yr}$ in almost all cases. Such standard deviations are not unusual, given the difficulties described; Ojha et al. (1994) arrived at values of $8 \mathrm{mas} / \mathrm{yr}$ for their standard deviation of the galaxy proper motions, which is consistent with our value, since their epoch difference is more than twice ours. The errors are almost as large as the values in all but a few cases. We nevertheless applied the galaxy calibration to put the proper motion in the extragalactic reference frame.

\subsection{The final sample}

The final sample consists of $114 \mathrm{sdB} / \mathrm{OB}$ stars. The observational data and data derived from observations, such as positions, $V$-magnitudes, distances, proper motions and radial velocities are shown in Table 1. This applies to all data, the new data and older data from the literature. The values of $\log g$ and $T_{\text {eff }}$ will be published in Edelmann et al. (2003).

\section{Kinematics and orbits}

\subsection{Calculating velocities and orbits}

The observational quantities $\alpha, \delta, d, \mu_{\alpha}, \mu_{\delta}, v_{\mathrm{rad}}-$ shown in Table 1 - are transformed into the $X, Y, Z, U, V, W$ system (for details, see Altmann \& de Boer 2000; de Boer et al. 1997). Additionally the orbital velocities $\Theta$ (the velocity component in the direction of Galactic rotation projected to the Galactic plane) and the velocities towards the Galactic centre $\Phi$, kinetic energies and angular momenta are calculated ${ }^{4}$. The resulting values for each star are represented in Table 2.

Furthermore we calculated orbits for the stars of our sample using the Galactic gravitational potential model of Allen \& Santillan (1991) backwards in time over 10 Gyr in steps of $1 \mathrm{Myr}$ (for more details see Altmann \& de Boer 2000).

From the shape of the orbits we derived the apo- and perigalactic distances, $R_{\mathrm{a}}$ and $R_{\mathrm{p}}$, and the eccentricity (ecc) given by

$e c c=\frac{R_{\mathrm{a}}-R_{\mathrm{p}}}{R_{\mathrm{a}}+R_{\mathrm{p}}}$

We also wish to consider the maximum distance a star reaches from the Galactic plane, $z_{\max }$. However, since the gravitational

\footnotetext{
${ }^{4}$ Because all of the stars are local $(d<5 \mathrm{kpc}), U$ and $W$ are quite similar to $\Phi$ and $\Theta$; however, especially for stars being well away from $Y=0 \mathrm{kpc}, \Theta$ and $\Phi$ become linear combinations of $U$ and $V$.
} 
Table 1. The observational data of the stars comprising our sample. The $B-V, V-R$ and $E_{B-V}$ values are only listed for those stars with new data.

\begin{tabular}{|c|c|c|c|c|c|c|c|c|c|c|c|}
\hline \multirow[t]{2}{*}{ Name } & \multicolumn{2}{|c|}{$\alpha(2000.0) \delta$} & \multirow[t]{2}{*}{ V } & \multirow{2}{*}{$\begin{array}{r}B-V \\
{[\mathrm{~m}}\end{array}$} & \multirow{2}{*}{$\begin{array}{l}V-R \\
\mathrm{~g}]\end{array}$} & \multirow[t]{2}{*}{$E_{B-V}$} & \multirow{2}{*}{$\begin{array}{r}d \\
{[\mathrm{kpc}]}\end{array}$} & \multirow{2}{*}{$\begin{array}{r}\mu_{\alpha} \cos \delta \\
{[\mathrm{mas} / \mathrm{y}}\end{array}$} & \multirow{2}{*}{$\begin{array}{l}\mu_{\delta} \\
\mathrm{r}]\end{array}$} & \multirow{2}{*}{$\begin{array}{r}v_{\mathrm{rad}} \\
{\left[\mathrm{km} \mathrm{s}^{-1}\right]} \\
\end{array}$} & \multirow[t]{2}{*}{ Source $^{a}$} \\
\hline & {$\left[{ }^{\mathrm{h} \mathrm{m} \mathrm{s}}\right]$} & {$\left[\begin{array}{lll}0 & 11\end{array}\right]$} & & & & & & & & & \\
\hline HE $0000-2355$ & 000322.06 & -233858.0 & 13.29 & -0.25 & -0.13 & 0.019 & 0.76 & +9.2 & -14.4 & -64 & \\
\hline HE 0001-2443 & 000431.00 & -242621.2 & 13.88 & -0.26 & -0.14 & 0.019 & 0.82 & +7.0 & -29.0 & +20 & \\
\hline HE 0004-2737 & 000646.26 & -272053.4 & 13.97 & -0.28 & -0.16 & 0.019 & 0.71 & +20.1 & -7.6 & +27 & \\
\hline PG 0004+133 & 000733.77 & +133557.6 & 13.06 & & & & 1.41 & +3.0 & -25.0 & -37 & $\mathrm{~B}+97$ \\
\hline HE 0021-2326 & 002359.33 & -230953.9 & 15.94 & -0.06 & +0.01 & 0.018 & 2.72 & +6.5 & -1.3 & -67 & \\
\hline HE 0031-2724 & 003353.89 & -270824.1 & 14.23 & -0.29 & -0.12 & 0.015 & 0.93 & -2.7 & +8.6 & -12 & \\
\hline PG 0039+049 & 004206.11 & +050923.4 & 12.88 & & & & 1.05 & +7.5 & -12.0 & +87 & $\mathrm{~B}+97$ \\
\hline CD -38 222 & 004258.31 & -380737.3 & 10.40 & & & 0.013 & 0.26 & +43.8 & -7.0 & -62 & HIP, AB00 \\
\hline HD 4539 & 004729.22 & +095855.7 & 10.31 & & & & 0.17 & +4.1 & +24.0 & +3 & HIP \\
\hline HE 0049-2928 & 005157.74 & -291207.5 & 15.78 & -0.22 & -0.12 & 0.014 & 2.20 & +15.4 & -17.5 & -15 & \\
\hline HE 0049-3059 & 005137.70 & -304256.2 & 14.41 & -0.24 & -0.13 & 0.017 & 1.22 & +16.9 & -2.1 & +52 & \\
\hline SB 410 & 010117.57 & -334245.4 & 12.63 & & & 0.020 & 0.54 & -11.0 & -12.9 & -56 & HIP \\
\hline Feige 11 & 010421.67 & +04 1337.3 & 12.06 & & & & 0.45 & +12.2 & -40.0 & +8 & $\mathrm{~B}+97, \mathrm{M}+99$ \\
\hline SB 459 & 010826.77 & -324311.6 & 12.30 & & & 0.023 & 0.44 & -7.7 & +1.7 & -24 & HIP \\
\hline HE 0123-2808 & 012533.35 & -275304.7 & 16.09 & -0.24 & -0.07 & 0.016 & 2.61 & +6.0 & -3.0 & +35 & \\
\hline HE $0127-4325$ & 012911.44 & -431027.8 & 14.60 & -0.23 & -0.13 & 0.018 & 1.71 & +8.3 & +7.9 & +16 & \\
\hline PG 0133+114 & 013626.26 & +113931.0 & 12.28 & & & & 0.77 & +20.7 & -34.4 & +6 & $\mathrm{M}+03$ \\
\hline PHL 1079 & 013826.93 & +033938.0 & 13.28 & & & & 0.81 & +11.1 & -17.9 & 0 & $\mathrm{~B}+97$ \\
\hline HE 0136-2758 & 013914.46 & -274321.8 & 16.17 & -0.23 & -0.14 & 0.021 & 2.20 & +15.6 & -22.9 & -159 & \\
\hline SB 707 & 014348.55 & -240510.2 & 11.77 & & & 0.015 & 0.26 & +88.4 & -46.2 & +30 & HIP \\
\hline PG $0142+148$ & 014539.57 & +1504 41.5 & 13.73 & & & & 1.17 & -17.4 & -0.4 & -131 & $\mathrm{~B}+97$ \\
\hline SB 744 & 014844.04 & -263612.8 & 12.32 & & & 0.016 & 0.46 & +90.6 & -47.0 & +27 & HIP, KHD, UB \\
\hline HE 0151-3919 & 015311.20 & -390418.0 & 14.31 & -0.22 & -0.10 & 0.014 & 2.11 & -7.4 & -43.0 & -176 & \\
\hline PG 0212+148 & 021511.08 & +150004.6 & 14.48 & & & & 1.75 & -3.8 & -9.2 & +50 & $\mathrm{~B}+97$ \\
\hline PG $0212+143$ & 021541.60 & +142918.0 & 14.58 & & & & 1.85 & +11.2 & -1.4 & +77 & $B+97$ \\
\hline HE 0218-3437 & 022059.75 & -342335.2 & 13.39 & -0.25 & -0.12 & 0.018 & 0.81 & +2.0 & -2.8 & +38 & \\
\hline HE $0218-4447$ & 022024.43 & -443328.5 & 12.89 & -0.28 & -0.14 & 0.016 & 0.64 & +47.9 & +1.7 & -15 & \\
\hline HE $0221-3250$ & 022358.15 & -323632.6 & 14.70 & -0.24 & -0.10 & 0.016 & 1.60 & +23.4 & -34.0 & -73 & \\
\hline HE 0230-4323 & 023254.68 & $\begin{array}{lll}-43 & 1027.8\end{array}$ & 13.78 & -0.22 & -0.11 & 0.023 & 1.03 & -11.7 & -9.2 & -102 & \\
\hline HE $0231-3441$ & 023400.25 & -342754.9 & 14.83 & -0.24 & -0.13 & 0.022 & 1.02 & +5.0 & +20.3 & -62 & \\
\hline PG $0242+132$ & 024538.86 & +132602.4 & 13.22 & & & & 1.39 & +17.2 & -9.7 & +11 & $\mathrm{~B}+97$ \\
\hline HE $0258-2158$ & 030017.80 & -214631.2 & 14.65 & -0.22 & -0.11 & 0.021 & 1.78 & -0.8 & +2.2 & +72 & \\
\hline HE 0307-4554 & 030925.93 & -454333.0 & 15.06 & -0.21 & -0.10 & 0.022 & 1.02 & +23.7 & -7.4 & -31 & \\
\hline HE 0315-4244 & 031747.12 & -423341.2 & 16.92 & -0.22 & -0.09 & 0.016 & 5.21 & +4.1 & -4.5 & +121 & \\
\hline HE 0324-3749 & 032614.98 & -251838.0 & 14.62 & -0.24 & -0.13 & 0.017 & 1.14 & -0.8 & -10.8 & +74 & \\
\hline HE 0340-3820 & 034247.06 & -381126.4 & 14.77 & -0.28 & -0.15 & 0.010 & 1.43 & +4.2 & +7.8 & -66 & \\
\hline HE 0341-2449 & 034336.35 & -243947.0 & 14.89 & -0.28 & -0.16 & 0.011 & 1.13 & +20.0 & -5.0 & +10 & \\
\hline PG 0342+026 & 034534.58 & +024752.8 & 10.94 & & & & 0.36 & +8.6 & -28.9 & +13 & TYC, PI \\
\hline HE $0343-4748$ & 034509.52 & -473854.0 & 14.19 & -0.17 & -0.07 & 0.006 & 2.12 & +5.8 & -2.7 & +44 & \\
\hline HE 0351-3536 & 035351.18 & -352735.3 & 14.11 & -0.23 & -0.11 & 0.009 & 0.86 & -0.6 & -8.2 & +64 & \\
\hline HE 0405-1719 & 040727.54 & -171115.5 & 14.00 & -0.28 & -0.14 & 0.025 & 0.91 & -2.6 & +15.8 & +70 & \\
\hline HE 0405-3839 & 040702.85 & -385146.1 & 14.39 & -0.26 & -0.12 & 0.007 & 1.47 & +5.2 & +2.8 & -44 & \\
\hline HE 0407-1956 & 041011.14 & -194853.6 & 13.61 & & & 0.030 & 0.96 & +13.9 & +41.7 & -6 & $\mathrm{~B}+92$ \\
\hline HE 0410-4901 & 041130.17 & -485348.0 & 14.51 & -0.21 & -0.06 & 0.017 & 1.71 & +5.4 & +10.7 & -27 & \\
\hline HE 0419-2538 & 042204.17 & -253100.5 & 13.67 & -0.23 & -0.12 & 0.044 & 1.29 & +4.1 & -8.2 & -29 & \\
\hline HE 0429-2448 & 043128.29 & -244156.8 & 15.30 & -0.24 & -0.12 & 0.048 & 1.18 & -5.6 & -9.0 & +15 & \\
\hline HE $0442-1746$ & 044434.88 & -174042.7 & 15.15 & -0.27 & -0.15 & 0.043 & 1.26 & -5.3 & +11.5 & +18 & \\
\hline
\end{tabular}


Table 1. continued.

\begin{tabular}{|c|c|c|c|c|c|c|c|c|c|c|c|}
\hline \multirow[t]{2}{*}{ Name } & \multicolumn{2}{|c|}{$\alpha(2000.0) \delta$} & \multirow[t]{2}{*}{ V } & $B-V$ & \multirow{2}{*}{$\begin{array}{l}V-R \\
\mathrm{~g}]\end{array}$} & \multirow[t]{2}{*}{$E_{B-V}$} & \multirow{2}{*}{$\begin{array}{r}d \\
{[\mathrm{kpc}]}\end{array}$} & $\mu_{\alpha} \cos \delta$ & $\mu_{\delta}$ & \multirow{2}{*}{$\begin{array}{r}v_{\mathrm{rad}} \\
{\left[\mathrm{km} \mathrm{s}^{-1}\right]}\end{array}$} & \multirow[t]{2}{*}{ Source $^{a}$} \\
\hline & {$\left[{ }^{\mathrm{h} \mathrm{m} \mathrm{s}}\right]$} & {$\left[{ }^{0},{ }^{\prime \prime}\right]$} & & {$[\mathrm{m}$} & & & & [mas/ & yr] & & \\
\hline HE 0444-4945 & 044614.09 & -494010.8 & 15.11 & -0.26 & -0.12 & 0.011 & 1.53 & +9.4 & +23.9 & +75 & \\
\hline HE 0447-3654 & 044915.62 & -364928.7 & 14.55 & -0.25 & -0.12 & 0.011 & 1.12 & +16.5 & -20.1 & +128 & \\
\hline HE 0452-3654 & 045352.66 & -364915.2 & 13.86 & -0.27 & -0.13 & 0.010 & 0.86 & +7.4 & +9.8 & -62 & \\
\hline HE 0500-3518 & 050231.67 & -351419.4 & 15.04 & -0.23 & -0.11 & 0.013 & 1.29 & +8.3 & +15.8 & +19 & \\
\hline HE 0504-2041 & 050639.67 & -203738.4 & 14.96 & -0.25 & -0.11 & 0.032 & 1.44 & +7.1 & +2.3 & -11 & \\
\hline HE 0505-2228 & 050747.46 & -222427.6 & 15.55 & -0.26 & -0.09 & 0.034 & 1.19 & +16.6 & +1.1 & -12 & \\
\hline HE 0505-3833 & 050658.85 & -382915.5 & 14.18 & -0.24 & -0.12 & 0.024 & 0.93 & +3.1 & +6.5 & +69 & \\
\hline HE $0510-4023$ & 051218.20 & -401934.5 & 14.84 & -0.22 & -0.08 & 0.048 & 1.51 & +3.3 & -6.2 & +49 & \\
\hline HE 0516-2311 & 051806.98 & -230845.2 & 15.60 & & & 0.037 & 2.27 & -17.9 & +17.8 & -12 & $\mathrm{HE}$ \\
\hline HE 0521-3914 & 052325.48 & -391154.3 & 15.55 & & & 0.026 & 1.82 & -15.0 & +11.2 & -87 & $K+99$ \\
\hline HE $0523-1831$ & 052531.32 & -182908.6 & 14.31 & -0.28 & -0.13 & 0.058 & 1.78 & +4.8 & -10.1 & -6 & \\
\hline HE $0532-4503$ & 053340.50 & -450135.3 & 16.06 & -0.22 & -0.11 & 0.037 & 2.56 & +8.2 & -2.7 & -166 & \\
\hline HE 0539-4246 & 054106.69 & -424531.9 & 14.80 & -0.20 & -0.11 & 0.045 & 1.28 & -0.2 & +3.7 & +71 & \\
\hline PG 0856+121 & 085902.72 & +115624.7 & 13.47 & & & & 0.99 & -19.4 & -19.8 & +85 & $\mathrm{~B}+97$ \\
\hline PG 0907+123 & 091007.60 & +120826.1 & 13.94 & & & & 1.52 & +6.4 & -2.6 & +85 & $\mathrm{~B}+97, \mathrm{M}+03$ \\
\hline PG $0918+029$ & 092128.23 & +024602.2 & 13.33 & & & & 1.04 & -28.5 & -20.0 & +104 & $\mathrm{~B}+97, \mathrm{TM}$ \\
\hline PG 0919+273 & 092239.83 & +270226.2 & 12.76 & & & & 0.35 & +22.9 & -19.8 & -65 & $B+97$ \\
\hline PG $1101+249$ & 110431.73 & +243944.8 & 12.79 & & & & 0.39 & -30.3 & +16.0 & -48 & $B+97$ \\
\hline PG 1114+073 & 111649.67 & +06 5930.8 & 13.06 & & & & 0.45 & -12.3 & -14.4 & +9 & $\mathrm{~B}+97, \mathrm{TM}$ \\
\hline PG 1232-136 & 123518.92 & -135509.3 & 13.34 & & & & 0.57 & -46.4 & -1.7 & +55 & $B+97$ \\
\hline PG $1233+427$ & 123551.64 & +422242.6 & 12.04 & & & & 0.32 & +3.6 & -18.1 & +61 & $B+97$ \\
\hline Feige 66 & 123723.52 & +250359.9 & 10.60 & & & & 0.18 & +2.7 & -26.7 & +1 & HIP, AB00 \\
\hline PG $1256+278$ & 125921.27 & +273405.2 & 14.25 & & & & 0.78 & -24.6 & +3.5 & +64 & $B+97$ \\
\hline PG 1343-101 & 134608.07 & -102648.2 & 13.76 & & & & 0.72 & -28.0 & -3.7 & +49 & $B+97$ \\
\hline HD 127493 & 143221.49 & -223925.6 & 10.04 & & & & 0.12 & -32.8 & -17.2 & +13 & HIP, AB00 \\
\hline PG $1432+004$ & 143519.83 & +00 1348.0 & 12.76 & & & & 0.76 & -9.4 & -25.8 & +1 & $\mathrm{~B}+97, \mathrm{TM}$ \\
\hline PG $1433+239$ & 143520.36 & +234527.5 & 12.45 & & & & 0.47 & -3.5 & -18.5 & -56 & $B+97$ \\
\hline PG 1452+198 & 5439.81 & +193700.9 & 12.48 & & & & 0.81 & -7.2 & -21.0 & +51 & $\mathrm{~B}+97$ \\
\hline PG 1519+640 & 152031.32 & +635208.0 & 12.46 & & & & 0.65 & +28.1 & +41.2 & +2 & TYC, TM \\
\hline PG $1619+522$ & 162038.74 & +520608.8 & 13.30 & & & & 0.77 & -3.6 & +9.0 & -52 & $\mathrm{~B}+97, \mathrm{M}+03$ \\
\hline HD 149382 & 163423.33 & -040052.0 & 8.87 & & & & 0.08 & -6.0 & -3.9 & +3 & HIP, AB00 \\
\hline PG $1647+252$ & 164908.97 & +251005.7 & 14.07 & & & & 0.71 & -3.8 & +12.3 & +26 & $B+97$ \\
\hline PG 1708+602 & 170915.90 & +601010.8 & 13.48 & & & & 1.79 & -14.9 & +12.1 & -8 & $\mathrm{~B}+97$ \\
\hline PG $1710+490$ & 171218.74 & +485835.9 & 12.90 & & & & 0.72 & +10.8 & -7.0 & -54 & $\mathrm{~B}+97, \mathrm{TM}$ \\
\hline PG $1716+426$ & 171803.54 & +423418.4 & 13.97 & & & & 1.20 & +7.1 & -21.8 & -4 & G98, M+03 \\
\hline PG $1722+286$ & 172411.97 & +283526.9 & 13.24 & & & & 0.87 & -4.0 & +10.0 & -34 & $\mathrm{~B}+97, \mathrm{TM}$ \\
\hline PG $1725+252$ & 172757.39 & +250835.7 & 12.89 & & & & 0.66 & -17.7 & +9.0 & -60 & $\mathrm{~B}+97, \mathrm{M}+03$ \\
\hline PG $1738+505$ & 173928.44 & +502925.1 & 13.15 & & & & 0.97 & -7.6 & +9.0 & +22 & $B+97$ \\
\hline UV 1758+36 & 180018.86 & +362856.3 & 11.37 & & & & 0.20 & -28.2 & +7.3 & 0 & HIP \\
\hline HD 171858 & 183756.68 & -231135.2 & 9.85 & & & & 0.16 & -15.0 & -24.7 & +74 & HIP, M+03 \\
\hline HE $2135-3749$ & 213840.59 & -373622.7 & 13.93 & -0.23 & -0.14 & 0.034 & 0.72 & +5.3 & +6.0 & -156 & \\
\hline HD 205805 & 213910.61 & -460551.5 & 10.16 & & & & 0.20 & +76.4 & -9.9 & -57 & HIP, B+97 \\
\hline HE $2154-4143$ & 215801.98 & -412849.7 & 15.17 & -0.25 & -0.12 & 0.019 & 1.49 & +5.3 & +5.7 & -15 & \\
\hline HE $2155-1724$ & 215815.92 & -170945.3 & 15.04 & -0.24 & -0.11 & 0.046 & 1.27 & +11.6 & -2.2 & -27 & \\
\hline HE $2156-1732$ & 215930.14 & -171821.6 & 14.45 & -0.22 & -0.08 & 0.041 & 1.39 & +8.7 & -10.2 & -77 & \\
\hline HE $2156-3927$ & 215935.50 & -39 1314.8 & 15.06 & -0.19 & -0.06 & 0.019 & 1.40 & +4.4 & +22.0 & -200 & \\
\hline HE $2201-2136$ & 220406.71 & -205909.3 & 15.90 & -0.23 & -0.11 & 0.038 & 1.88 & +9.2 & -3.9 & -23 & \\
\hline PG 2204+035 & 220716.50 & +034219.8 & 14.25 & & & & 1.18 & +7.5 & -6.0 & +81 & $\mathrm{~B}+97$ \\
\hline
\end{tabular}


Table 1. continued.

\begin{tabular}{|c|c|c|c|c|c|c|c|c|c|c|c|}
\hline \multirow[t]{2}{*}{ Name } & \multicolumn{2}{|c|}{$\alpha(2000.0) \delta$} & \multirow[t]{2}{*}{ V } & $B-V$ & \multirow[t]{2}{*}{$V-R$} & \multirow[t]{2}{*}{$E_{B-V}$} & \multirow{2}{*}{$\begin{array}{r}d \\
{[\mathrm{kpc}]}\end{array}$} & \multirow{2}{*}{\multicolumn{2}{|c|}{$\begin{array}{c}\mu_{\alpha} \cos \delta \quad \mu_{\delta} \\
{[\mathrm{mas} / \mathrm{yr}]}\end{array}$}} & \multirow{2}{*}{$\begin{array}{r}v_{\text {rad }} \\
{\left[\mathrm{km} \mathrm{s}^{-1}\right]}\end{array}$} & \multirow[t]{2}{*}{ Source $^{a}$} \\
\hline & {$\left[{ }^{\mathrm{h} \mathrm{m} \mathrm{s}}\right]$} & {$\left[\begin{array}{lll}0 & \prime \prime & \prime \prime\end{array}\right]$} & & {$[\mathrm{m}$} & & & & & & & \\
\hline HE 2205-1952 & 220841.30 & -193739.4 & 14.59 & -0.28 & -0.14 & 0.023 & 1.01 & +7.5 & -8.8 & -52 & \\
\hline HE $2213-4158$ & 21617.70 & -414322.2 & 16.15 & -0.34 & -0.12 & 0.012 & 3.95 & +5.6 & +10.3 & -4 & \\
\hline PG 22 & 222124.83 & +021618.6 & 14.17 & & & & 1.15 & +1.1 & -11.8 & +21 & $\mathrm{~B}+97$ \\
\hline HE $2222-3738$ & 222456.43 & -372330.2 & 14.89 & -0.23 & -0.13 & 0.016 & 1.39 & +32.0 & -6.0 & -134 & \\
\hline PG 2226+094 & 222858.41 & +093721.8 & 14.08 & & & & 1.13 & +14.3 & +0.4 & -48 & $\mathrm{~B}+97, \mathrm{TM}$ \\
\hline PG 2259+1 & 230145.82 & +133837.5 & 14.48 & & & & 1.38 & +0.6 & -9.6 & +16 & $\mathrm{~B}+97$ \\
\hline Feige $108^{b}$ & 231612.41 & -015034.5 & 12.96 & & & & 0.40 & -7.8 & -16.5 & +40 & $\mathrm{~B}+97$ \\
\hline Feige $109^{b}$ & 231726.89 & +075204.9 & 13.76 & & & & 1.13 & -1.2 & +8.1 & -37 & $\mathrm{~B}+97, \mathrm{TM}$ \\
\hline PG $2337+070^{b}$ & 234004.83 & +07 1711.0 & 13.47 & & & & 0.77 & -19.1 & -37.4 & -27 & $\mathrm{~B}+97$ \\
\hline HE 23 & 234015.33 & -292759.8 & 14.45 & -0.25 & -0.15 & 0.019 & 0.87 & +14.3 & +8.4 & -26 & \\
\hline HE 2340-2806 & 234241.39 & -275001.6 & 15.01 & -0.16 & -0.05 & 0.017 & 1.46 & +1.7 & +11.3 & -20 & \\
\hline SB 815 & 234422.00 & -342700.4 & 10.96 & & & 0.013 & 0.25 & -22.3 & -7.2 & +24 & HIP \\
\hline HE $2343-2944$ & 234617.75 & -292749.9 & 15.06 & -0.21 & -0.13 & 0.019 & 1.29 & +5.5 & -15.2 & -0 & \\
\hline HE 2349-3135 & 235143.64 & -311852.9 & 15.91 & -0.28 & -0.11 & 0.014 & 2.08 & -6.6 & -1.9 & +180 & \\
\hline PG $2349+002^{b}$ & 235153.26 & +002818.0 & 13.28 & & & & 0.82 & -10.1 & -15.7 & -84 & $\mathrm{~B}+97$ \\
\hline SB 884 & 235236.10 & -30 1009.1 & 12.09 & & & 0.019 & 0.37 & +30.4 & -13.2 & -3 & HIP \\
\hline HE $2355-3221$ & 235822.47 & -320439.1 & 15.18 & -0.22 & -0.14 & 0.012 & 1.69 & -0.1 & -15.5 & +61 & \\
\hline PG 2358+107 & 000106.73 & +110036.3 & 13.62 & & & & 0.83 & -3.0 & -14.0 & -19 & $\mathrm{~B}+97$ \\
\hline HE 2359-2844 & 000138.46 & -282742.8 & 16.62 & & & 0.019 & 1.53 & +7.8 & -12.0 & -129 & $\mathrm{~L}+00$ \\
\hline
\end{tabular}

${ }^{a}$ Data sources: unmarked, data from this work and from Edelmann et al. (2003), B+97=de Boer et al. (1997), AB00=Altmann \& de Boer (2000), HIP = ESA (1997). Additional sources: Photometry: HE = Hamburg-Eso-Survey, K+99= Koen et al. $(1999)$, B+92= Beers et al. (1992), L+00 = Lamontagne et al. (2000). Spectroscopy $\left(\log g, T_{\text {eff }}, v_{\text {rad }}\right)$ : TM= Marsh (priv. comm.), M+99= Moran et al. (1999), M+03 = Morales-Rueda et al. (2003), PI = Moehler et al. (1990), KHD = Kilkenny et al. (1988), UB = Unglaub \& Bues (1990). 4. Astrometry: TYC $=$ Høg et al. (2000), G98 = Geffert (1998).

${ }^{b}$ de Boer et al. (1997), but revised values for the proper motions.

potential diminishes at larger galactocentric distance, $\varpi$, we calculated

$n z e=\frac{z_{\max }}{\varpi\left(z_{\max }\right)}$,

the normalised $z$-extent of the orbit, which is more relevant than $z_{\max }$ alone. The eccentricities and nze are also shown in Table 2.

Finally we stress that we calculated the orbits for the long timespan of 10 Gyr only because it gives a better representation of the orbits' shapes. This applies in particular to low velocity Halo type stars taking a long time to complete one revolution. The reader should be aware that orbits are subject to gradual change over time due to gravitational interactions with local high density areas in the Galaxy. Therefore the orbit calculated over a certain time does not represent the true trajectory in the far past.

The errors of the velocity components depend on the uncertainties of the input values, namely $10 \%$ for the distances, $30 \mathrm{~km} \mathrm{~s}^{-1}$ for the radial velocities and $5 \mathrm{mas} / \mathrm{yr}$ for the proper motions (which means an error in tangential velocity of about $30 \mathrm{~km} \mathrm{~s}^{-1}$ at $1.5 \mathrm{kpc}$ ). For a star at a typical distance of $1.5 \mathrm{kpc}$ this results in an error estimate of $35 \mathrm{~km} \mathrm{~s}^{-1}$. Stars at lower distance have a smaller error in the tangential velocity while objects further away have larger errors.
Those stars with Hipparcos data, or with available systemic radial velocities have far smaller errors than indicated in this error estimation. In contrast to this, stars in undetected close binary systems (see Sect. 2.3.1), could have far more uncertain radial velocities.

\subsection{Analysis of the velocities and velocity dispersions}

For the majority of the objects, the orbital velocities have values which are similar to those of disk stars. This has been found before by other studies, e.g. de Boer et al. (1997) or Thejll et al. (1997). However a minor portion of the sample has orbital velocities that are significantly below or above those expected for disk stars (see Fig. 2); some stars have even a near zero or even slightly negative orbital velocity. As seen in earlier studies, the majority of the sdB stars have velocities rather indicative of disk orbits, but with $\bar{\Theta}$ somewhat lower than that for Thin Disk stars, while the velocity dispersions are larger (see Table 3 ).

$\sigma_{V}$ is larger than expected for the Thick Disk alone, reflecting the fact that the Halo and Thin Disk components are present in our sdB sample. A significant old Thin Disk contribution can also be seen by looking at $\bar{\Theta}$ which is higher than what most studies of Thick Disk kinematics arrive at (see e.g. Ojha et al. 1994). However, due to the composition of the sample, which 
Table 2. Positions, velocities (given in the Galactic euclidic system $X Y Z U V W$ and $\Phi, \Theta$ ) as well as the angular momentum $I_{z}$ and morphological orbital data $\left(R_{\mathrm{a}}, R_{\mathrm{p}}, z_{\max }\right.$ and eccentricity $(e c c)$, normalised $z$-extent (nze), see text) of all stars.




Table 2. continued.

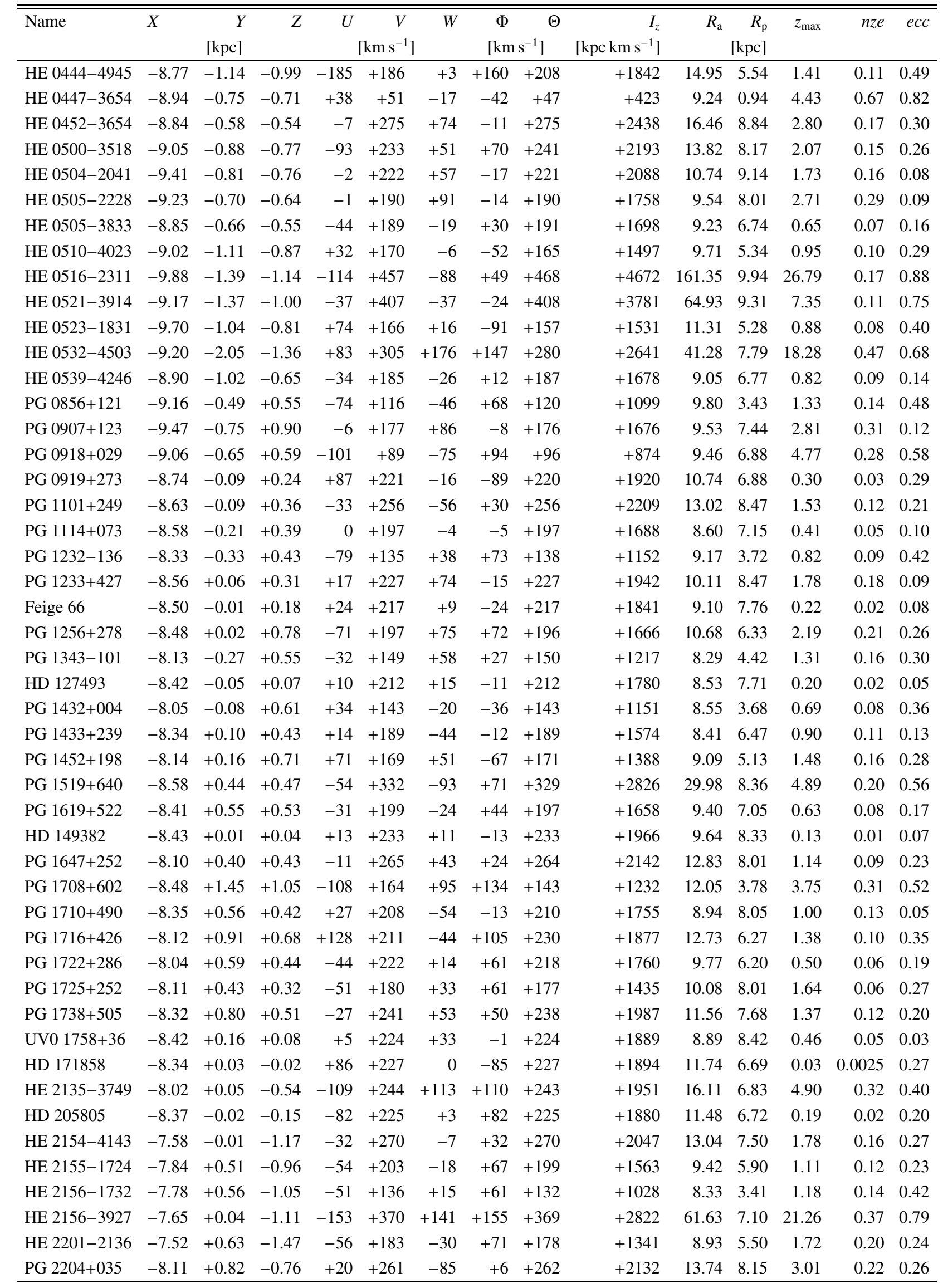


Table 2. continued.

\begin{tabular}{|c|c|c|c|c|c|c|c|c|c|c|c|c|c|c|}
\hline Name & $X$ & $\begin{array}{r}Y \\
{[\mathrm{kpc}]}\end{array}$ & $Z$ & $U$ & $\begin{array}{r}V \\
\left.\mathrm{~km} \mathrm{~s}^{-1}\right]\end{array}$ & $W$ & $\begin{array}{c}\Phi \\
{[\mathrm{km}}\end{array}$ & ${ }_{1} \Theta$ & $\begin{array}{r}I_{z} \\
{\left[\mathrm{kpc} \mathrm{km} \mathrm{s}^{-1}\right]}\end{array}$ & $R_{\mathrm{a}}$ & $\begin{array}{r}R_{\mathrm{p}} \\
{[\mathrm{kpc}]}\end{array}$ & $z_{\max }$ & $n z e$ & $e c c$ \\
\hline HE 2205-1952 & -8.00 & +0.36 & -0.80 & -31 & +172 & +19 & +38 & +170 & +1363 & 8.33 & 5.12 & 0.92 & 0.11 & 0.24 \\
\hline HE 2213-4158 & -6.25 & -0.08 & -3.25 & -104 & +406 & -71 & +99 & +407 & +2544 & 62.40 & 7.02 & 25.40 & 0.44 & 0.80 \\
\hline PG $2218+020$ & -8.16 & +0.76 & -0.80 & +43 & +201 & -41 & -24 & +205 & +1676 & 8.56 & 7.27 & 1.21 & 0.14 & 0.08 \\
\hline HE $2222-3738$ & -7.76 & +0.07 & -1.17 & -227 & +147 & +12 & +228 & +145 & +1123 & 16.53 & 2.89 & 2.55 & 0.16 & 0.70 \\
\hline PG 2226+094 & -8.27 & +0.84 & -0.72 & -64 & +184 & 0 & +83 & +177 & +1468 & 12.30 & 7.17 & 1.53 & 0.08 & 0.32 \\
\hline PG 2259+134 & -8.43 & +1.03 & -0.91 & +39 & +20 & -44 & -14 & +212 & +1803 & 8.79 & 8.22 & 1.38 & 0.16 & 0.03 \\
\hline Feige 109 & -8.46 & +0.75 & -0.84 & -7 & +24 & +63 & +29 & +240 & +2033 & 12.50 & 8.38 & 1.85 & 0.19 & 0.16 \\
\hline Feige 108 & -8.45 & +0.22 & -0.33 & +43 & +238 & -33 & -37 & +239 & +2022 & 10.95 & 8.05 & 0.75 & 0.07 & 0.15 \\
\hline PG 2337-070 & -8.53 & +0.48 & -0.60 & +138 & +154 & -28 & +129 & +162 & +1381 & 11.60 & 4.32 & 1.12 & 0.10 & 0.46 \\
\hline HE 2337-2944 & -8.28 & +0.08 & -0.84 & -63 & +241 & +16 & +65 & +240 & +1986 & 11.96 & 7.42 & 1.22 & 0.10 & 0.23 \\
\hline HE 234 & -8.16 & +0.17 & -1.40 & -38 & +298 & +24 & +44 & +298 & +2428 & 18.82 & 8.09 & 3.09 & 0.17 & 0.40 \\
\hline SB 815 & -8.43 & +0.00 & -0.24 & +44 & +238 & -7 & -43 & +238 & +2009 & 10.89 & 7.86 & 0.30 & 0.03 & 0.16 \\
\hline HE 2343-2944 & -8.20 & +0.11 & -1.25 & +20 & +136 & +2 & -18 & +137 & +1120 & 8.31 & 3.88 & 1.26 & 0.15 & 0.36 \\
\hline HE 2349-3135 & -8.02 & +0.10 & -2.02 & +117 & +254 & -151 & +114 & +256 & +2050 & 22.52 & 7.68 & 11.84 & 0.61 & 0.49 \\
\hline PG 2349+002 & -8.52 & +0.42 & -0.70 & +76 & +163 & +61 & -68 & +167 & +1422 & 9.69 & 5.16 & 1.62 & 0.17 & 0.31 \\
\hline SB 884 & -8.42 & +0.02 & -0.36 & -28 & +191 & -1 & +28 & +191 & +1605 & 8.69 & 6.34 & 0.37 & 0.04 & 0.16 \\
\hline HE 2355-3221 & -8.14 & +0.04 & -1.65 & +78 & +126 & -43 & -77 & +126 & +1026 & 9.00 & 3.52 & 2.33 & 0.26 & 0.44 \\
\hline PG 2358+107 & -8.62 & +0.52 & -0.63 & +49 & +193 & -9 & -37 & +196 & +1691 & 9.18 & 6.73 & 0.69 & 0.08 & 0.15 \\
\hline HE 2359-2844 & -8.23 & +0.12 & -1.50 & -23 & +122 & +125 & +24 & +122 & +1002 & 8.35 & 4.79 & 4.92 & 0.70 & 0.27 \\
\hline
\end{tabular}

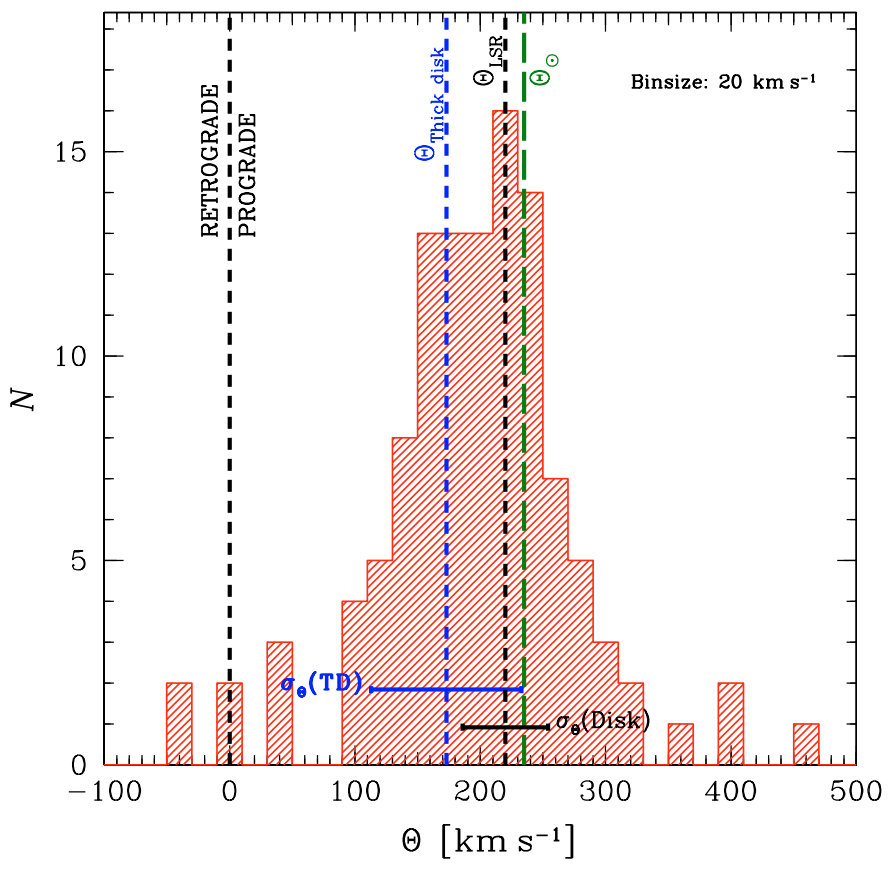

Fig. 2. Histogram of the orbital velocities $\Theta$ for all 110 stars of the sample. The values for $\Theta_{\text {Thick disk }}$ and $\sigma_{\Theta}(\mathrm{TD}$, Disk) have been taken from Ojha et al. (1994).

is certainly lacking stars currently located at low $z$-heights, we miss a fraction of the Thin Disk stars (see Sect. 2.1.1).

The stars with large $\Theta$ (between 250 and $300 \mathrm{~km} \mathrm{~s}^{-1}$ ) are also evident in the Toomre diagram (Fig. 3), which shows the kinematic divergence of a sample of stars. In our case most data points are located within $v_{\text {pec }} \leq 100 \mathrm{~km} \mathrm{~s}^{-1}$, with the region of $v_{\text {pec }} \leq 150 \mathrm{~km} \mathrm{~s}^{-1}$ also well populated for $\Theta \leq \Theta_{\mathrm{LSR}}$. This obvious asymmetry shows a behaviour usually known as asymmetric drift. It means that kinematically hotter populations tend to rotate slower than kinematically cooler populations. The reason for this effect lies in the greater eccentricity of the orbits of such objects and will be discussed in greater detail in the analysis of the kinematic behaviour over the whole orbit (Sect. 3.3.2). A few points lie further out, indicating a kinematic behaviour quite different from the rest of our sdB stars. The central concentration is well filled to $\Theta \simeq 300 \mathrm{~km} \mathrm{~s}^{-1}$. At very low peculiar velocities $\left(v_{\text {pec }} \simeq 30 \mathrm{~km} \mathrm{~s}^{-1}\right)$ only relatively few points are present.

The Bottlinger diagrams (Fig. 4) show a concentration of stars at low values of $\Phi$ and $W$ respectively near $\Theta_{\text {LSR }}$. However the concentration of data points does appear to be slightly shifted in respect to the $\Phi, W=0$ axis. In both panels (but especially in the one showing $W, \Theta$ ) the points seem to be somewhat inhomogeneously distributed. Again a few stars deviate from the general concentration by a large degree.

The stars of our sample show a behaviour which is kinematically hotter than but not too different from that of the Sun. This implies that the majority of our stars belong to the Thick Disk because their orbital velocities are somewhat lower than those of stars with solar kinematics. A few stars have orbital velocities which differ a lot from those of the rest, either being far higher or lower than those of the rest. Two or perhaps three stars even have mildly retrograde orbits. These are presumably not disk stars but members of a non-rotating Halo population. Whether the stars having a high velocity and those with 
Table 3. $U V W, \Theta \Phi$ velocities, angular momentum, eccentricities and $n z e$ with their dispersions for the 110 star sample.

\begin{tabular}{lrrrrrrrrrrrrrrrrrrr}
\hline Subsample & $N$ & $\bar{U}$ & $\sigma_{U}$ & $\bar{V}$ & $\sigma_{V}$ & $\begin{array}{c}\bar{W} \\
{\left[\mathrm{~km} \mathrm{~s}^{-1}\right]}\end{array}$ & $\sigma_{W}$ & $\bar{\Theta}$ & $\sigma_{\Theta}$ & $\bar{\Phi}$ & $\sigma_{\Phi}$ & $\begin{array}{c}\bar{I}_{\mathrm{z}} \\
{\left[\mathrm{kpc} \mathrm{km} \mathrm{s}^{-1}\right]}\end{array}$ & $\sigma_{I_{\mathrm{z}}}$ & $e \bar{c} c$ & $\sigma_{e c c}$ & $n \bar{z} e$ & $\sigma_{n z e}$ \\
\hline all & 114 & -8 & 74 & +198 & 79 & +12 & 64 & +198 & 80 & +6 & 74 & +1700 & 705 & 0.33 & 0.22 & 0.51 & 2.74 \\
$R<8.5 \mathrm{kpc}$ & 52 & -13 & 60 & +208 & 61 & +10 & 53 & +208 & 61 & +22 & 62 & +1698 & 465 & 0.28 & 0.18 & 0.18 & 0.42 \\
$R>8.5 \mathrm{kpc}$ & 62 & -3 & 70 & +196 & 84 & +13 & 72 & +196 & 85 & -7 & 79 & +1761 & 902 & 0.35 & 0.23 & 0.30 & 0.49 \\
$e c c<0.55$ & 99 & -5 & 59 & +198 & 52 & +10 & 55 & +199 & 53 & +5 & 58 & +1707 & 460 & 0.26 & 0.13 & 0.19 & 0.36 \\
$e c c>0.55$ & 15 & -29 & 136 & +196 & 172 & +31 & 102 & +193 & 68 & +15 & 138 & +1655 & 1947 & 0.78 & 0.13 & 2.57 & 7.15 \\
$Z<0.25 \mathrm{kpc}$ & 10 & +23 & 46 & +225 & 12 & 0 & 24 & +225 & 12 & -23 & 47 & +1909 & 97 & 0.15 & 0.09 & 0.03 & 0.03 \\
$Z>0.25 \mathrm{kpc}$ & 104 & -11 & 75 & +195 & 82 & +14 & 66 & +195 & 84 & +9 & 75 & +1680 & 738 & 0.34 & 0.22 & 0.55 & 2.86 \\
\hline Sun & 1 & +10 & - & +235 & - & +8 & - & +235 & - & -10 & - & +1998 & - & 0.08 & - & 0.01 & - \\
\hline
\end{tabular}

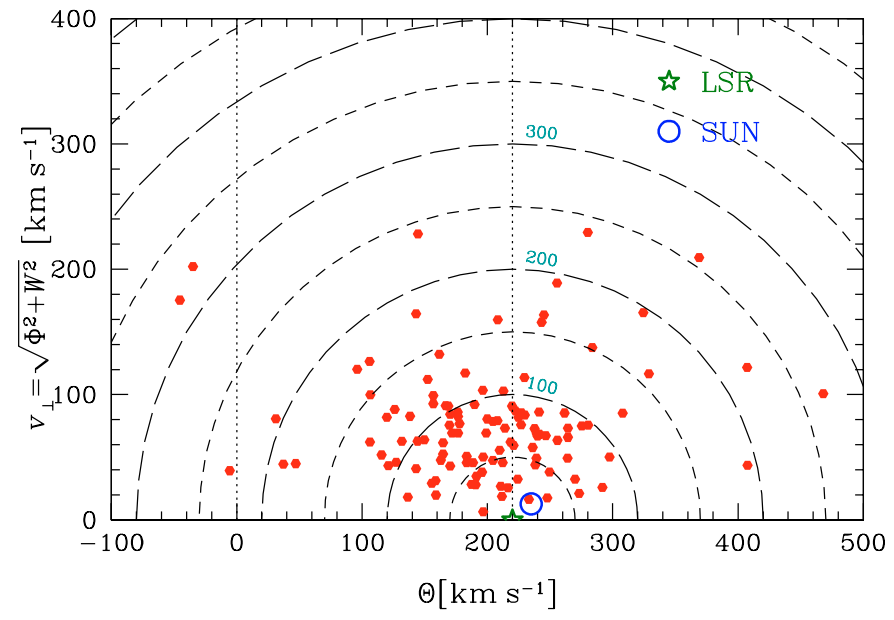

Fig. 3. Toomre diagram $(\Theta$ versus velocity perpendicular to the Galactic plane) showing the kinematic divergence of the stars of our sample. The circles with values in $\mathrm{km} \mathrm{s}^{-1}$ indicate $v_{\text {pec }}=$ $\sqrt{\Phi^{2}+W^{2}+\left(\Theta-\Theta_{\mathrm{LSR}}\right)^{2}}$. Note the asymmetry in the central condensation of data points with $v_{\text {pec }}<150 \mathrm{~km} \mathrm{~s}^{-1}$. A star denotes the LSR and a circle the Sun's values.

a low $\Theta$ are of similar or different origin will be discussed in the next section, when the orbits are examined. This also applies to those disk stars which also have relatively high $\Theta$ values.

In order to find differences in kinematics in parts of our sample and to make a crude populationary separation we applied simple cuts to the sample. The normal way to achieve this is by using a non kinematic selection criterion such as metallicity which is not possible in our case (see Sect. 1). For this reason we have to use kinematical criteria which unfortunately means introducing biases. First of all we divided our sample with simple criteria (Table 3 ). Cuts were made at $R=8.5 \mathrm{kpc}$, $e c c=0.55$ and $z=0.25 \mathrm{kpc}$, to roughly separate Thin Disk, Thick Disk or Halo. However all populations samples created with these simple cuts still show contamination of other populations. The two Disk components could not be reliably separated, while Disk and Halo seem to be generally well separated.

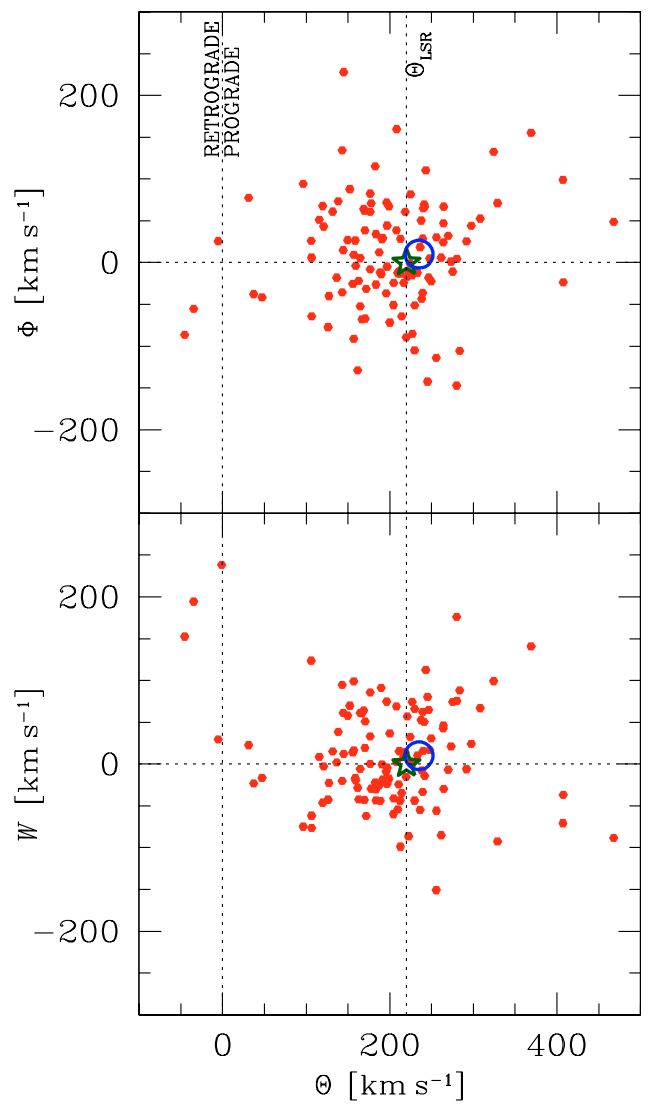

Fig. 4. Bottlinger and $\Theta-W$ diagrams of the stars of our sample show the orbital velocity plotted against the two other components, the velocity towards the Galactic centre ( $\Phi$, top) and perpendicular to the Galactic plane ( $W$, bottom). A star denotes the LSR and a circle the Sun's values.

\subsection{The orbits}

\subsubsection{Orbit morphology}

The morphologies of the orbits show large varieties and we show some exemplary orbits in Fig. 5. However most show box type orbits typical of Disk/Thick Disk stars. Six stars have chaotic orbits, or semi chaotic orbits. These are the stars venturing very close to the Galactic centre, like the majority of the HBA stars of Altmann \& de Boer (2000). About the same number of stars have orbits going to very large galactocentric 


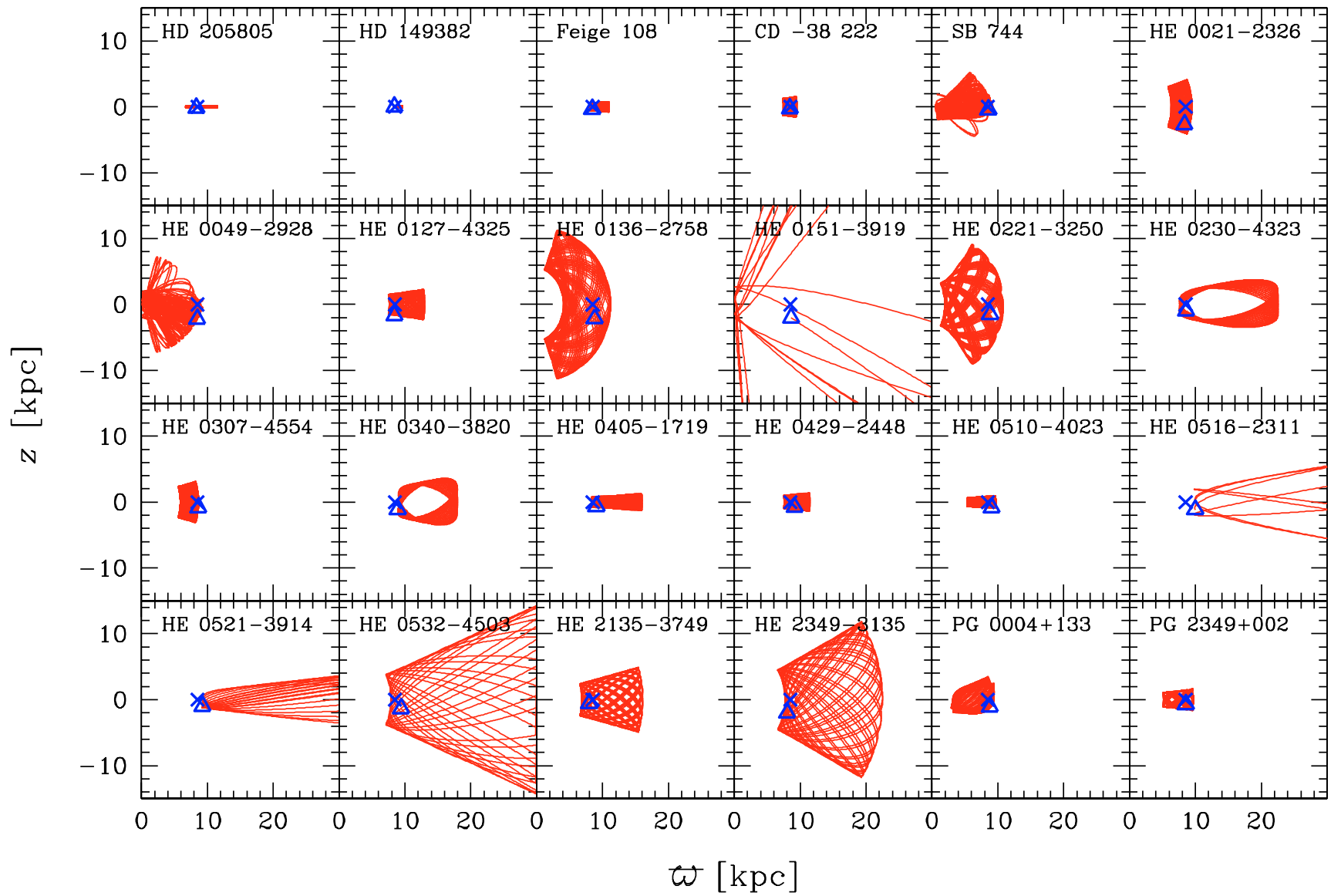

Fig. 5. Examples of orbits of sdB stars. The orbits are depicted as meridional plots and calculated over a timespan of $10 \mathrm{Gyr}$. The triangle denotes the current position of each star and the cross the current position of the Sun. Note that this figure does not attempt to be representative of the sample but to show all types of orbits of our stars. Stars with Halo type orbits are overrepresented in this plot. For the orbit of the Sun (being a Thin Disk star) see Fig. 1 of de Boer et al. (1997).

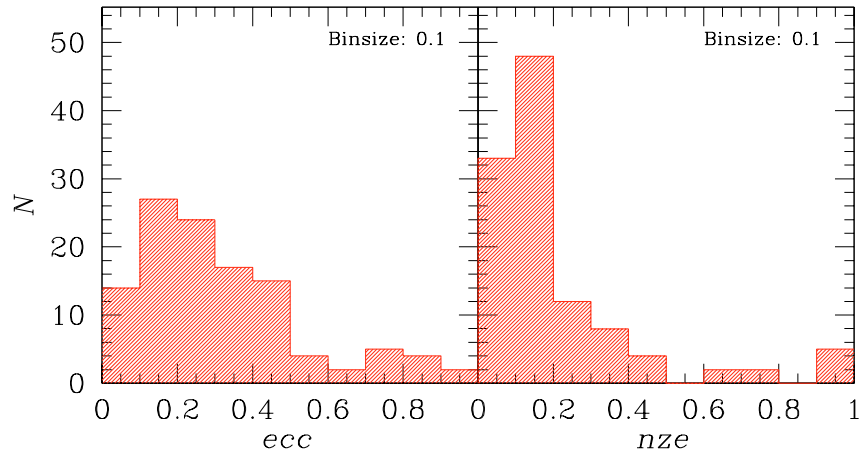

Fig. 6. Histograms showing the distributions of ecc (left panel) and nze (right panel) for the stars of the sample. Note the peaks at low values in ecc and nze and the local minimum in the distribution of the eccentricities near $e c c=0.55$.

distances, one having its apogalacticon at $\sim 160 \mathrm{kpc}$. These are the stars which have an orbital velocity much higher than that of the LSR, in some cases approaching the escape velocity of the Milky Way.

The stars have orbits with eccentricities (see Fig. 6, left panel) spanning almost the complete range, however more than $80 \%$ have $e c c<0.5$. This is the region mainly populated by Disk and Thick Disk stars. A minority has ecc $>0.7$ with the intermediate zone somewhat underpopulated. This might mean that the sdBs are part of two kinematically quite distinct groups, namely one with orbits of small to medium eccentricity and another having very eccentric orbits.

The distribution of the normalised $z$-extents of our sample (see Fig. 6, right panel) is more peaked than that of the values for ecc, almost all of the stars having $n z e \leq 0.4$, but there is a long tail to high values. We do not see a separation at intermediate nze values. However this is expected as nze is, amongst others, a measure for the inclination of an orbit. A group of stars all having small orbit inclinations will show up as a large peak at low values of nze in a histogram. In contrast to that, a group of stars having orbits with more random inclinations will populate the range of $n z e$ without any preferential value (except in the case of stellar streams or moving groups). This is basically what can be seen in Fig. 6, namely a peak of a population of low nze stars and a level distribution of a group of stars with a large spread in orbit inclination.

\subsubsection{Analysis of the kinematics over the whole orbits}

The analysis of the current velocities (Sect. 3.2) gives important information about the kinematical behaviour and population membership of the sample. However velocities are in 


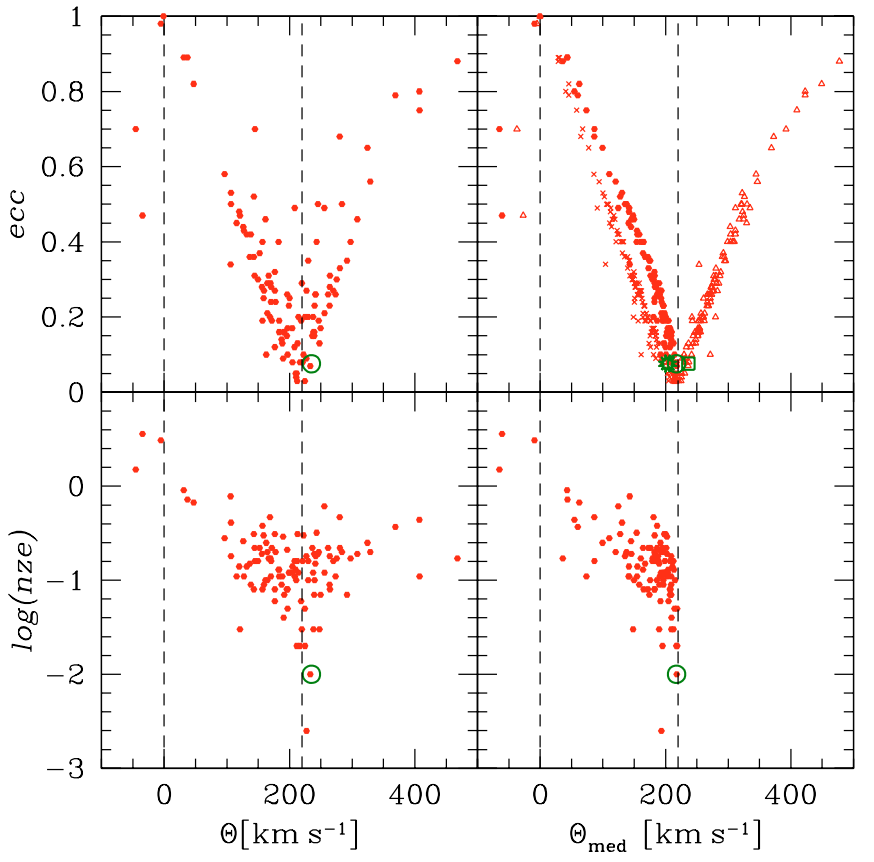

Fig. 7. Plots $\Theta$ against $e c c$ and $\log n z e$ : On the left side the current values of $\Theta$ are plotted, on the right side the median $\Theta$. In the upper right panel the maximum (open triangles) and minimum (crosses) $\Theta$ in the orbit are plotted as well. The Sun's values are represented by the open circles and in the upper right panel as the open square and star. $\log n z e$ is used rather than $n z e$ to gain access to more detail at low values. The dashed lines indicate the border between prograde and retrograde motion and the $\Theta_{\mathrm{LSR}}$ at $220 \mathrm{~km} \mathrm{~s}^{-1}$.

general not conserved quantities, and therefore change over time. Analysing a sample of stars solves this problem because mean values of the velocity components and their dispersions are conserved quantities. However they possibly suffer from selection effects. For example stars have, during a revolution around the Galactic centre, a considerable range in orbital velocity $\Theta$ (e.g. the Sun has a $\Theta_{\min }$ of $203 \mathrm{~km} \mathrm{~s}^{-1}$ and a $\Theta_{\max }$ of $\left.237 \mathrm{~km} \mathrm{~s}^{-1}\right)$.

Furthermore, all stars spend most of the time at galactocentric distances $(\varpi)$ near the turning points of the orbit, i.e. near the peri- and especially apogalactic distance. The second Keplerian law and the stellar density law of our Galaxy then leads to the result that the high velocity part of a sample is less populated than the low velocity end. For a sample of stars this means that the distribution of time related velocities is not necessarily a Gaussian but may be a broader, possibly even a bimodal distribution.

Another consequence of the second Keplerian law is the asymmetric drift, i.e. the effect that a sample of stars generally stays behind the local standard of rest. This lagging behind depends on the eccentricity of a star's orbit; the more eccentric it is the more the mean orbital velocity deviates from $\Theta_{\mathrm{LSR}}$, an effect clearly seen in Fig. 7. For this reason a sample of stars with moderately eccentric orbits is on average slower in Galactic rotation than a sample of stars with near circular orbits. The Toomre diagram (Fig. 3) shows this effect especially well in the asymmetric distribution of data points, with more objects being at $\Theta<\Theta_{\text {LSR }}$ than at $\Theta>\Theta_{\text {LSR }}$.

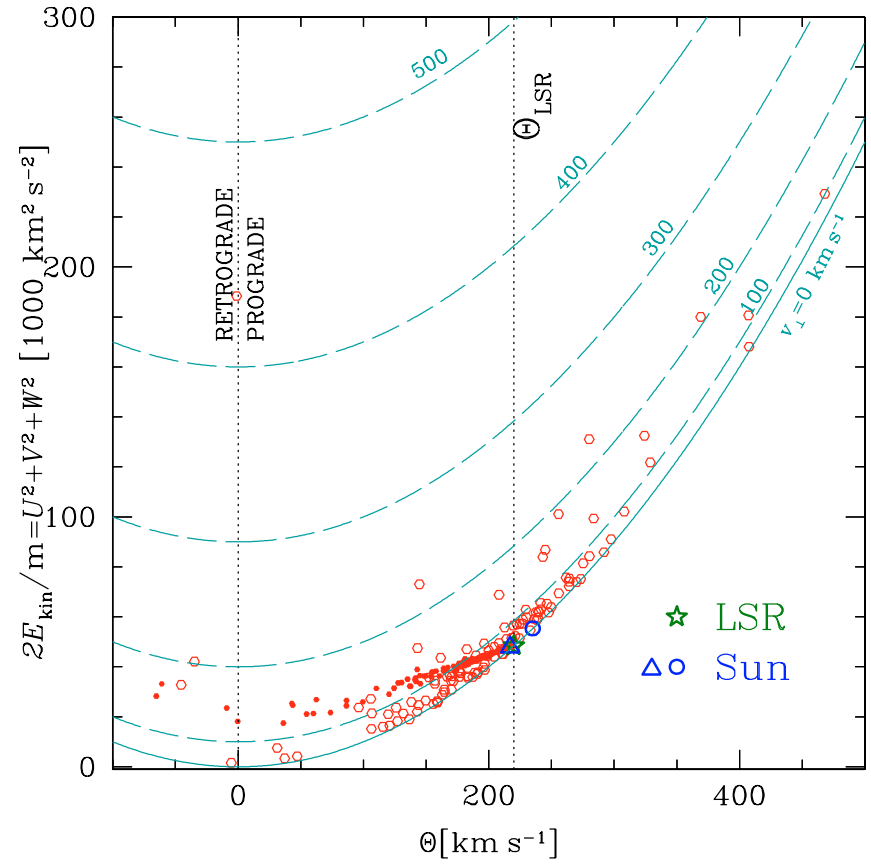

Fig. 8. Diagram of $\Theta$ as well as $\Theta_{\text {med }}$ against the total kinetic energy $\left(E_{\text {kin }}\right)$. Open symbols represent current values of $\Theta$ and $E_{\text {kin }}$, smaller filled hexagons medianised values $\left(\Theta_{\text {med }}, E_{\text {kin,med }}\right)$. The parabolic curves denote lines of equal orthogonal velocity $\left(v_{\perp}\right.$, the velocity perpendicular to $\Theta$ ). Most of the stars cluster around the LSR along the low $v_{\perp}$. The medianised values lie on an almost straight line pointing from the LSR towards the lower left. The LSR is marked by a star, the Sun's current values by a circle, its medianised one by a triangle.

In Sect. 3.2 we noted that there are several stars with relatively high orbital velocities $\left(\Theta \geq 250 \mathrm{~km} \mathrm{~s}^{-1}\right)$. Looking at the values of $\Theta$ along the complete orbits of these stars shows that the kinematics is very similar to that of stars having $\Theta$ of less than $200 \mathrm{~km} \mathrm{~s}^{-1}$. This means that every sample of Thick Disk stars must have a number of objects having a $\Theta$ significantly faster than the LSR (for solar like orbits the range in $\Theta$ covered is much smaller). Now looking again at Fig. 2, one sees that the histogram peaks at $\sim 220 \mathrm{~km} \mathrm{~s}^{-1}$ and has a plateau down to $150 \mathrm{~km} \mathrm{~s}^{-1}$. The reason for this could be that the stars come from two populations, namely a kinematically hotter one, i.e. the Thick Disk, and a minority of stars, having much tighter orbits, representing the Thin Disk.

In Fig. 7 we plotted the current and median values of the orbital velocities $\Theta$ against the eccentricities and normalised $z$-extents of the stars. In the upper left panel of Fig. 7 one can see that most of the stars have low to moderate eccentricity orbits but a group has high $e c c$ values with a less populated region near $e c c=0.6$ (which was already evident in the histogram of Fig. 6). Moreover it is apparent that while there are more stars having a $\Theta$ smaller than $\Theta_{\text {LSR }}$, about $1 / 4$ of the objects have higher velocities. As $\Theta$ of the stars changes over time we plotted the median of $\Theta$ over the whole orbit ${ }^{5}$.

\footnotetext{
5 We took the median rather than the mean because it separates the values a little more, and for all except the highly eccentric orbits there is only a minor difference between both values.
} 
To show the complete range of variation in $\Theta$ we also plotted the maximum and minimum values in the upper right panel of Fig. 7. The $\Theta_{\text {med }}$ all lie on a line, except those of the retrograde orbits. Some deviate a little from this line. These are stars on somewhat more inclined orbits having a higher $W$ velocity component (which also adds to the velocity supporting the orbit; a star on a higher inclined orbit can have a less eccentric orbit with the same orbital velocity, because of its larger $W$ velocity component). Again, the two groups of stars can be seen as well as the division at $e c c=0.55$. At $e c c<0.2$ the trend in $\Theta_{\text {med }}$ is small, and at higher values it gets more pronounced. Again the Thin Disk part, if it exists, makes up a large part of this low ecc group.

In the lower two panels of Fig. 7 plots of $\log n z e(\log n z e$ because most values of $n z e$ are clumped together at low values) against $\Theta, \Theta_{\text {med }}$ are shown. The left panel shows the distribution of nze of our sample. The central condensation marks the majority of disk stars, and the outliers towards low or very high $\Theta$ are the Halo stars. The spur towards low $n z e$ at LSR velocities consists of datapoints of solar type orbits.

On the plot $\log n z e$ versus $\Theta_{\text {med }}$ the different subdivisions of our sample can be seen even better, on the left are the Halo stars, which have a low $\Theta_{\text {med }}$ (the high velocity Halo stars lie in the far left of this diagram). The bulk of the Thick Disk stars cluster around $\Theta_{\text {med }}=200 \mathrm{~km} \mathrm{~s}^{-1}, \log n z e=-0.9$, and the solar kinematics extension protrudes towards the bottom of the diagram. At values of $\log n z e>-0.7$ there seems to be a gap appearing between $\Theta_{\mathrm{LSR}}$ and the data points. It is possible that the Thin Disk population represented by the spur more or less ends at this point (which corresponds to a $z_{\max }$ of $\sim 1.7 \mathrm{kpc}$ or 5-6 Thin Disk scale heights). The stars having a $\Theta_{\text {med }}$ of more than about $190 \mathrm{~km} \mathrm{~s}^{-1}$ as well as $n z e$ of less than $\sim 0.2(\log n z e=-0.7)$ are the prime candidates for the Thin Disk component.

So potentially the lower right plot of Fig. 7 is a suitable tool to kinematically separate Thin and Thick disk stars, if these populations are kinematically detached at all.

Another method of analysing the kinematics of stars is using the kinetic energy (or total velocity). In Fig. 8 we plotted the kinetic energy $2 \cdot E_{\text {kin }} / m=U^{2}+V^{2}+W^{2}$ against $\Theta$. The parabolas plotted in Fig. 8 are lines of equal $v_{\perp}$ (velocity orthogonal to $\Theta$ ). This velocity is, together with $\Theta-\Theta_{\text {LSR }}$ a measure of kinetic temperature, the higher its value the more an object's orbit deviates from a circular orbit. For low values of $v_{\perp}$, the deviation from $\Theta_{\mathrm{LSR}}$ gives information about the kinetic temperature. As can be easily seen, most of the values cluster around the LSR on a banana shaped region alongside the $v_{\perp}=0 \mathrm{~km} \mathrm{~s}^{-1}$ isovelocity line. This clustering means that the majority of them is kinematically relatively cool. A few stars are located further away, and in some cases quite far away from the $v_{\perp}=0 \mathrm{~km} \mathrm{~s}^{-1}$ contour. These are the kinematically hot stars. Another reason that most of our stars have quite low $v_{\perp}$ values is that most stars are near their orbital turning points (see the top of this subsection), i.e. their apo- or perigalacticon. In these orbital phases the $\Phi$ component is minimised.

As the current $\Theta$ and $E_{\text {kin }}$ are just snapshot values, we also calculated the median of both quantities using the whole orbit and plotted these in Fig. 8 as well. These data points lie nicely on a line pointing towards lower $\Theta$ and lower $E_{\text {kin }}$ away from the LSR. There is a gap near $\Theta=110 \mathrm{~km} \mathrm{~s}^{-1}$. Stars located to the left of this gap have rather hot orbits. Their considerable dispersion in inclination is shown by the spread along the line (caused by the $W$ velocity component). They represent the Halo population. The stars at $\Theta>110 \mathrm{~km} \mathrm{~s}^{-1}$ are the Disk stars. Clearly the asymmetric drift of each star can be seen. The warmer an orbit is kinematically (seen by increasing $v_{\perp}$ ), the lower its orbital velocity is.

Considering $E_{\text {kin }}$ and $\Theta_{\text {med }}$ in Fig. 8, there is (similar to what we saw in Figs. 6 and 7) a clear division between the Halo and Thick Disk with the division line here being at $\Theta=$ $110 \mathrm{~km} \mathrm{~s}^{-1}$. Dividing Thick and Thin Disk is not so straightforward. Perhaps only the distribution of a sample complete to the Galactic plane will lead to a separation of these two kinematical populations.

To conclude, analysing the kinematics of the whole orbits instead of just the current velocity gives further insight into the kinematic behaviour of a group of stars. In our case the Halo and Disk components of our sample are clearly discerned (Figs. 6-8). Of the 114 stars, 16 (14\%) belong to the hitherto unknown Halo group, the rest are Disk stars ${ }^{6}$. Thin and Thick Disk groups cannot really be separated, at least not with the current sample, which is perhaps lacking in Thin Disk stars. Possibly the kinematics of both disk components are not really disjunct so that for a detailed analysis statistically complete distributions are required.

\section{Determining a scale height for the stars using their orbits}

\section{1. z-probability plot and scale height}

We have derived the $z$-distribution ${ }^{7}$ of our sample of 114 stars using the orbits of the stars, as done before in de Boer et al. (1997) for 41 stars. The program used to calculate the orbits does so for a fixed time per step, in our case 1 Myr. Plotting a histogram of the orbit in $\varpi$ or $z$ leads to the $\varpi$ resp. $z$ probability distribution for the star, i.e. the probability to find the star at a certain $\varpi$ or $z$ distance. Plotting the histogram for the whole sample (by adding up the individual histograms) leads to the probability distribution for the complete group. From this the $z$-density gradient of the sample can be deduced. One may now

\footnotetext{
${ }^{6}$ A further candidate may be HE 2349-3135, which, while having $\Theta=256 \mathrm{~km} \mathrm{~s}^{-1}$ and an orbit eccentricity of 0.49 (these values would classify it as a Disk star), travels to $12 \mathrm{kpc}$ above the Galactic plane. This is rather typical for Halo stars. Therefore its status is unclear; we do not count it as a Halo star when stating the percentage of Halo stars, but do include it in our kinetically selected Halo sample which is also used for the scale height determination.

${ }^{7} z$ is the (positive) distance between the Galactic plane and a point (such as a star) while $Z$ means the $Z$-coordinate of the point in the $X Y Z, U V W$ system. Technically we are determining the $Z$-probability distribution, because we are measuring the slope above and below the Galactic plane, i.e. for $Z<0 \mathrm{kpc}$ and $Z>0 \mathrm{kpc}$. Assuming the symmetry of the disk in $Z$ direction both slopes will have similar values and can therefore be averaged to one, namely the $z$-distribution.
} 


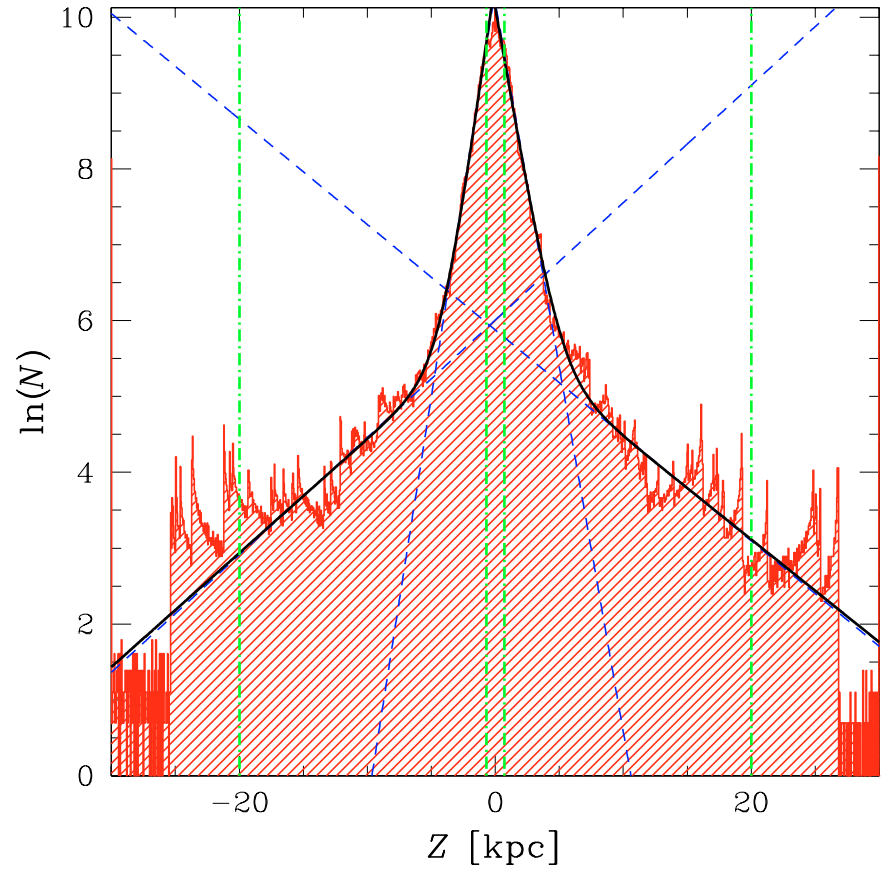

Fig. 9. Histogram of the $z$-distance statistics of all the stars of the sample. The logarithmic distribution clearly shows the two components (see also Sect.4.1). Fits using data within the dash-dotted verticals lead to the scale heights as given in Table 4 . The binsize is $50 \mathrm{pc}$.

fit an exponential distribution and determine the scale height using the relation

$\ln N(z)=\ln N_{0}-\frac{z}{z_{0}}$,

with $N(z)$ being the number density at $z, N_{0}$ being the density at $z=0 \mathrm{kpc}$, and $z_{0}$ the scale height. The scale height is the reciprocal value of the slope of the $\ln N$ distribution. As we do not have a defined volume in which the stars are located we are unable to determine absolute values for $N(z)$ and $N_{0}$. What we can determine, however, are relative values of the form $N_{1}(0) / N_{2}(0)$ in the case that there are two or more slopes.

The method is described in greater detail in de Boer et al. (1997). The $z$-distribution is depicted in logarithmic form in Fig. 9.

Figure 9 clearly shows that our distribution consists of two components with different $z$-distributions, a central one with a steep slope and an outer shallow distribution. Fitting linear equations to the various regions leads us to scale heights of $1.0( \pm 0.1) \mathrm{kpc}$ for the central (steep) part and $7( \pm 2) \mathrm{kpc}$ for the outer (shallow) parts. For the fit of the steep slope we used a fitting interval of $[0.7,4]$ and $[-4,-0.7] \mathrm{kpc}$ and $[7,17]$ resp. $[-18,-7] \mathrm{kpc}$ for the shallow slope ${ }^{8}$. The results for the upper $(Z>0 \mathrm{kpc})$ and lower $(Z<0 \mathrm{kpc})$ half of the distribution are: for the central part:

$z_{0,+}=1.04 \mathrm{kpc}, z_{0,-}=0.93 \mathrm{kpc}$

\footnotetext{
8 The intervals used for this component are slightly different because of a disturbing spike in the one direction, which would somewhat falsify the fit result.
}

Table 4. Compilation of our results for the scale heights $\left(z_{0}\right)$ and midplane densities $N_{0}$ for Thick Disk and Halo. The mid-plane density of the Halo is presented as the density ratio of Halo and Thick Disk.

\begin{tabular}{lccc}
\hline \hline Method & Thick Disk & \multicolumn{2}{c}{ Halo } \\
& $z_{0}$ & $N_{0}$ & $z_{0}$ \\
& {$[\mathrm{kpc}]$} & {$[\%]$} & {$[\mathrm{kpc}]$} \\
\hline separate linear equations & 0.98 & 1.3 & 6.8 \\
2-component fit & 0.93 & 1.2 & 7.0 \\
adopted values $^{a}$ & 0.93 & 1.2 & 7.0 \\
\hline
\end{tabular}

${ }^{a}$ These values rely on the 2-component fit.

for the outer parts:

$z_{0,+}=7.2 \mathrm{kpc}, z_{0,-}=6.5 \mathrm{kpc}$.

The main reason for the greater uncertainty for the outer part is that this relies on only a small group of stars and therefore suffers from small number statistics. On the whole the result for the component with the steep slope is very similar to that of de Boer et al. (1997) based on a sample only $1 / 3$ of the size of the present. The other component was not discernible for de Boer et al. (1997), because their sample had only few data points at $z>2 \mathrm{kpc}$.

For small $z$ the distribution is less well known. Here selection effects come into play (see Sect. 2.1.1).

\subsection{Effects of errors}

The effects of the errors of the input parameters, i.e. distance, radial velocity, and proper motion on the derived scale height was analysed by de Boer et al. (1997). They added errors to these quantities, and then computed the scale heights anew. The most important effect may be a systematic error in the distances: the scale height would decrease if the distances were systematically too small, and vice versa. The effects of the other quantities are smaller, with the scale height being smallest when no error is added. As we expect similar effects on our sample, we did not repeat the error analysis but refer to de Boer et al. (1997).

\subsection{Scale height and galactocentric distance}

To see how the scale height results vary with galactocentric distance we cut the cumulative orbit file of our whole sample in bins in galactocentric distance $(\varpi)$ and determined the scale heights for each. In all bins (except the outermost bin $\varpi \geq 15 \mathrm{kpc}$, where the inner, steep distribution is missing) both components were found; however the outer, shallow component was overwhelmed by the steep component and thus hard to identify in the bins near $\varpi \simeq 8.5 \mathrm{kpc}$. The results for the scale height of the steep component is only marginally smaller than the result derived from all data points. Several aspects have to be taken into account here such as the increase in scale height with $\varpi$ and the increased number of solar like orbits in the central bins, two aspects which counteract each other because the latter orbits have a smaller vertical extent. Therefore no significant trend with $\varpi$ can be seen in the steep scale height. 
The extended, high $z$ distribution is based on only a few stars. For this reason one does expect a large spread in the derived scale heights of the subsamples. Apart from this there is a trend from small to large scale heights with $\varpi$. This is an effect of the diminishing gravitational potential with galactocentric distance.

\subsection{Robustness of the scale heights, separating the different populations}

In order to test the stability of the values of our scale heights we made tests with different fit interval limits. Furthermore, to determine how the various components influence each other, we derived scale heights with various subsamples excluded.

Shifting the lower and upper border of the fit interval of the steep component leads to a variation of the result of about $100 \mathrm{pc}$. The choice of interval width used for fitting leads to an error of $\sim 50 \mathrm{pc}$, which means that the derived slope and hence scale height is relatively robust in this respect. The fit intervals used to determine the slope have a larger influence on the Halo component because of various peaks caused by individual stars.

The presence of the Halo component has some influence on the scale height obtained for the Thick Disk. Without it (selected using the results of Sect. 3) the scale height tends to be $100-150 \mathrm{pc}$ lower. For the complete disk sample without the 17 stars classified as Halo members, we find a scale height of the steep component of $0.84 \pm 0.1 \mathrm{kpc}$ instead of the $0.98 \pm 0.1 \mathrm{kpc}$ for the whole sample. For this reason the Halo component should be removed before calculating the scale height of the Thick Disk.

Removing the stars with the coolest, i.e. most solar-like, kinematics (thus excluding stars with $e c c<0.15$ and $n z e<$ $0.15)$ leads to a slightly higher scale height of just over $0.9 \mathrm{kpc}$. Obviously the influence of Thin Disk stars is much lower. This becomes quite clear, when one considers that removing stars with a nze lower than a certain value only changes the distribution in the middle. Therefore, the selection effects described in Sect.2.1.1 do not play a significant role for the determination of the scale height. If the sample were complete for the low $z$ stars, the central part would fill in and possibly result in a third perhaps still steeper component, representing the Thin Disk.

The end points of the fit are more significant for the Halo component than for the disk part. This is expected as spikes in the distribution caused by individual stars are important this subsample contains only 17 stars. On the other hand the influence of the disk on the Halo scale height is small.

Another approach is to fit a 2-component function to this. This double exponential function (which accounts for the overlap in the region where both components are of similar strength) has the following form:

$\ln N(z)=\ln \left(N_{\mathrm{D}, 0} \cdot \mathrm{e}^{-z / z_{\mathrm{D}}}+N_{\mathrm{H}, 0} \cdot \mathrm{e}^{-z / z_{\mathrm{H}}}\right)$

with the indices $\mathrm{D}, \mathrm{H}$ referring to the Disk and Halo respectively. The resulting fit of this equation to the data is shown in Fig. 9. Here we used fitting intervals of $[0.7,17] \mathrm{kpc}$ and $[-18,-0.7] \mathrm{kpc}$ (shown in Fig. 9). Varying the fitting intervals lead to the following results: Raising the lower limits causes the scaleheight to slowly rise. This trend was mainly caused by one side of the distribution, the other one did not change. The upper limit has the same difficulties caused by the spikes as in the single exponential fits.

The resulting values for the scale heights are:

$z_{\mathrm{D}}=0.93 \pm 0.09 \mathrm{kpc}$

$z_{\mathrm{H}}=7.0 \pm 0.5 \mathrm{kpc}$

This means that there is a difference of $0.09 \mathrm{kpc}$ between the Disk scale heights derived using population separation and the 2-component fit. The difference between the two values for the Halo is as large as $0.5 \mathrm{kpc}$; this may be related to the effect we see for the Disk scale height, but as the values for the Halo are not very certain and depend much on the fit intervals, we do not further discuss the differences of the Halo scale height values. Reasons for this small discrepancy could lie in the fit, as the presence of the Halo component may have an influence on the derived value for the Disk, fitting intervals could still play a role here.

To conclude, we consider the 2-component method the most trustworthy and finalise the value of the scale heights as: $0.93 \pm 0.08 \mathrm{kpc}$ for the Disk (or $0.9 \pm 0.1 \mathrm{kpc}$ ), $7.0 \pm 1.0 \mathrm{kpc}$ for the Halo component ${ }^{9}$.

\subsection{Midplane number ratio of the two components found}

The steep distribution has a peak value of $\ln N_{0}=10.3$ and the shallow part peaks at $\ln N_{0}=6.5$. Therefore the relative density of the shallow with respect to the steep component is $1.25 \pm 0.25 \%$.

The zero point of the broad distribution has a large uncertainty because it relies on the data of relatively few objects. Therefore the ratio of densities is only an estimate.

\subsection{Discussion of results}

Our scale height study resulted in finding two components, one with a large and one with a moderate scale height. A compilation of the results with the different strategies described in the sections above, is given in Table 4. The latter scale height is with $z_{0}=0.9 \mathrm{kpc}$ very similar to that found for the sdB stars by de Boer et al. (1997). It is also similar to the determinations of the scale height of the Thick Disk (see e.g. Ojha 1994; Kerber et al. 2001; Chen et al. 2001 and discussion below). Thus the steep component in Fig. 9 can be identified with the Galactic Thick Disk population.

The analysis of the kinematics (Sect. 3) showed that there are some stars with solar kinematics. These stand out as an extra double peak in the centre of the linear distribution - also

\footnotetext{
9 This error is an estimate, but certainly more appropriate than the values derived from the difference of the two fits (upper and lower half) given above. The value for the Halo part varies more than the $0.5 \mathrm{kpc}$ when fitted under different fitting circumstances.
} 
vaguely seen near the peak of the logarithmic histogram of Fig. 9. Presumably these and perhaps some more belong to the Old Thin Disk.

The shallow, high $z$ component represents a drastically different population of stars. It is rather hard to imagine a population with such a scale height to be disk-like if one considers the Galaxy's radius being of the order $15 \mathrm{kpc}$. So we speculate that this component is actually a spheroid or an ellipsoid. To make significant quantitative statements about the shape of the distribution of this population more stars are needed than the few which are discussed here. But this subsample clearly consists of members of the Halo.

The density ratio of the Halo to the Disk component extrapolated to the Galactic plane is $1.2 \%$ (see Table 4). This value is of course quite uncertain. Kerber et al. (2001) found a density ratio of $0.2 \%$ for Halo and Thin Disk. Chen et al. (2001) found a local relative density of the Halo against the Thin Disk of $0.125 \%$, in total values between $0.05 \%$ and $0.4 \%$ are found. Our higher values for the Halo to Disk density is probably due to the fact that our Disk stars are rather members of the Thick than the Thin Disk (or a mixture of both). The literature values for the Thick/Thin Disk density ratio range from about $5 \%$ to $10 \%$. Assuming now a mean Thick/Thin Disk relative density of $7.5 \%$, and a Halo/Thin Disk ration of $0.15 \%$ (see above) the Halo/Thick Disk ratio becomes approximately $2.0 \%$. This is - given the large spread in the literature values - rather similar to the value we derived from the sdB stars. Therefore the production rate of $\mathrm{sdB}$ stars seems to be of equal magnitude in both populations. Given the inherent different nature of stars in both Galactic components, such as metallicity, we conclude that these do not play a large role in the formation process of sdB stars. For the Thin Disk we can unfortunately not make any definite statement about the production rate of sdB stars in respect to the other populations.

\section{Discussion: kinematics and the population membership of sdB stars}

As shown in Sects. 3 and 4 the sdB stars of our sample belong to different populations. In this section we want to discuss the different groups and implications for the evolutionary processes that lead to the formation of $\mathrm{sdB}$ stars and questions of Galactic structure.

\subsection{Kinematical biases}

Apart from the selection effects discussed in Sect. 2.1.1 which are mainly caused by the sample composition, there are other effects resulting from the kinematics and orbital morphologies themselves. These therefore not only occur in studies of specific object types but in any kinematic study such as the one presented here.

Stars with different vectors of motion (in respect to the Sun) have different probabilities of venturing into the vicinity of the Sun (defined by the observable range of this study). An object with a motion differing a lot from the solar motion will only stay in the solar vicinity for a short while; a star with a solar like orbit will remain there for a very long time. One the other hand an object of the "fast moving" type (i.e. relative to the Sun) has a greater probability of moving into the solar vicinity, because a "solar like" object, once it is outside the solar range, will stay outside for a long time. Presumably these two effects ${ }^{10}$ compensate each other or nearly do so; therefore we do not expect significant skewness in our analyses caused by this. However stars with extreme kinematics might very well be affected. This especially applies to stars on retrograde orbits or in principle to stars orbiting significantly faster than the Sun.

A similar phenomenon could occur when looking at the motion perpendicular to the Galactic plane. Here stars on polar orbits are presumably underrepresented in respect to stars travelling on an orbit with a smaller inclination angle. Therefore spherical populations such as the Halo may appear flattened somewhat when examining the distributions of their stars using the kinematics. The effect on disk-like populations such as the Disks should be of much smaller magnitude. Therefore we do not take it into account in the present study.

We do not quantitatively analyse probable effects caused by the selection effects discussed in this subsection, because the focus of this study was to determine quantities like the scale height of the Disk, which is not as much affected by such a bias, and to find a possible Halo population. The number of Halo stars is too small to give more than rather crude values for the scale height and other quantities. However we do point out that in the future (when yet larger samples give us the opportunity of a more detailed analysis of the Halo population) it is certainly worthwhile if not essential to analyse and quantify the biases introduced by such phenomena.

\subsection{The Disk}

The vast majority of the sdB stars was found to belong to the Galactic Disk. While most stars have orbits of moderate eccentricity and reach normalised $z$-heights $(n z e)$ of around 0.2 , there are quite a few which have near solar kinematics, and are therefore more likely to be associated with the Thin Disk. Unfortunately, the sample composition prevents us from separating the two populations unambiguously if these are separate at all (see Sect. 2.1.1). The histogram in Fig. 2 shows a Thick Disk-like distribution with a Thin Disk peak. This implies that the stars of our sample come from both Disk components. Because of our result for the scale height and the velocity dispersions, we conclude that the majority of the sample belongs to the Thick Disk.

On the whole most other studies arrive at values similar to what we found for the scale height and kinematics of the Thick Disk. There is however some disagreement among these studies concerning the scale height and hence the local relative density. Kerber et al. (2001) derive a scale height for the Thick Disk of between 0.8 and $1.2 \mathrm{kpc}$. Reylé \& Robin (2001) favour a value of $0.8 \mathrm{kpc}$, Chen et al. (2001) arrive at $0.58-0.75 \mathrm{kpc}$ but with a much higher local density of $6.5-13 \%$ of the density of the Thick Disk. In contrast to those results,

\footnotetext{
10 This is in principle the old problem of the two clocks: Which one is better, a clock being slow (or fast) one minute per hour or one that is completely broken?
} 
Reid \& Majewski (1993) find a scale height of $1.4 \mathrm{kpc}$, in this case the local density being not large, $\sim 2 \%$. As can be seen the values differ by at least a factor of 2 . Our value of $1 \mathrm{kpc}$ is only a little above the values obtained in most studies.

The velocities and velocity dispersions are under much less dispute than the scale height. Most studies arrive at values near those of Ojha et al. (1994), i.e. $\sim 50 \mathrm{~km} \mathrm{~s}^{-1}$ for each $U V W$ component and a value of $175 \mathrm{~km} \mathrm{~s}^{-1}$ for the mean orbital velocity of the Thick Disk. Our larger value for $\bar{\Theta}$ and $\sigma_{\Theta}$ is related to the Thin Disk and Halo contamination. When we only consider stars with ecc $\leq 0.55$ thus excluding most of the Halo objects, we obtain values for $\sigma_{\Theta}$ of about 50 to $60 \mathrm{~km} \mathrm{~s}^{-1}$ (see Table 3).

\subsection{The Halo}

As described in Sects. 3 and 4, the Halo component stars can relatively easily be separated from the Disk population. Of the complete sample of 114 stars, 16 (see Sect.3.3.2) or $\sim 15 \%$ form our Halo sample. It is approximately divided in half, one part having small orbital velocities, i.e. having orbits similar to those of the HB stars of Altmann \& de Boer (2000), and the other, the high velocity component, having $\Theta$ velocities far larger than that of the LSR. As can be seen in Fig. 8 there is no or only a small difference between the kinematics of the two groups. They represent orbits in different phases - the low velocity ones are currently near their apogalacticon and the others are close to the perigalacticon where $\Theta$ is highest. Therefore the orbits of both groups of the stars of our sample cover different radial spaces in the Milky Way. That they form a bimodal velocity distribution rather than a continuum in our sample may be a consequence of the selection effects discussed in Sect. 5.1 and the small number statistics.

However some of the orbits of the high velocity group extend very far out, further out than what is generally considered to be the limits of the Galaxy. Their orbits are rather transient than bound orbits. So one might speculate that the history of these stars is somewhat different than that of the low velocity Halo stars.

It has long been known that stellar groups exist at distances large compared with what is regarded as the normal extent of the Milky Way. Harris (1996) lists 9 globular clusters with a current galactocentric distance of more than $30 \mathrm{kpc}$, the most remote objects being at $100 \mathrm{kpc}$ or more. Dinescu et al. (1999a) have 6 objects in their sample of 38 globulars with kinematic data which go beyond $30 \mathrm{kpc}$. With one object (Pal 3) in common that makes 14 globular clusters of the 147 listed in Harris (1996), venturing that far. However not only globular clusters but also field stars have been found that far from the Galactic centre. Vivas et al. (2001) found 150 RR Lyrae stars at distances of about $50 \mathrm{kpc}$ and Yanny et al. (2000) found a large number of HBA stars forming a group or a stream at a similar distance.

One of the Sun's neighbours, Barnards star ${ }^{11}$ features an orbit very similar to that of the stars of our "high velocity Halo". It travels to an apogalactic distance of $36 \mathrm{kpc}$, and has a $\Theta$

\footnotetext{
11 Orbit calculated from Hipparcos data.
}

of $367 \mathrm{~km} \mathrm{~s}^{-1}$. So clearly this remote spatial regime is not unpopulated.

Our group of Halo and high velocity Halo stars is too small to make definite statements about the origin and behaviour of distant stellar groups as a whole. However we speculate that two of our stars might have a common origin because their trajectories are quite similar and extreme. Analysing a larger sample might therefore give insight into moving streams of Halo stars which are being incorporated into our Galaxy (see e.g. Helmi \& White 1999).

\subsection{Aspects of the stellar evolution history of sdB stars}

Apart from questions concerning the structure of the Galaxy, there are still aspects concerning the evolution of stars to sdB stars which have not yet been satisfactorily solved. This especially applies to their extreme mass loss leaving a He-core nearly completely stripped of all hydrogen. It has been suspected for a long time that a large part, or all, of the sdB stars are in fact products of binary evolution (see e.g. Iben \& Tutukov 1987), with their unusually thin H-shell being the result of mass transfer from the evolving primary (the star later turning into the sdB star). In fact many sdBs show a secondary component in their spectrum and also in their colour indices (see e.g. Thejll et al. 1994; Theissen et al. 1995; Aznar Cuadrado \& Jeffery 2002). As described in Sect. 2.3.1 many, however by far not all (or even the majority) of the apparently single sdB stars have radial velocity variations revealing an unseen companion, such as a low mass main sequence star or a white dwarf. This means that it is certainly not clear whether close binary evolution is the only way sdB stars are formed.

A kinematical study does not as such prove or disprove a theory about stellar evolution. However our results show that sdBs occur in all locally observable older populations rather than in one alone. This means that it is unlikely that there are factors such as metallicity in play. Furthermore sdBs form from stars of a quite significant spread in mass and hence in age, and the formation times of Halo, Thick and Thin Disk are of ages differing by several Gyr. Therefore our results provide at least some support for the binary scenario.

Dorman et al. (1993) have calculated models of horizontal branches for various metallicities ranging from very metalpoor to supersolar metallicity. These show a thinning out of the occupation of the HB in the middle, increasing with metallicity. D'Cruz et al. (1996) have further enhanced these models, and tried to find an explanation for the extreme mass loss required to make HBB and sdB stars. The models were calculated for masses of around $1 M_{\odot}$ and somewhat less massive for the metal-poor stars, taking into account that Halo stars are generally assumed to be older than the more metal-rich stars of the Thick Disk and even more than those with solar metallicity.

Our present work shows that sdB stars can be found in all populations. The earlier work (Altmann \& de Boer 2000), analysing the kinematics of all types of HB-stars in the temperature range between the RR-Lyrae and the sdB regimes, 
came to the result that very few if any HBA stars are Disk stars (with relatively high metallicities) and that only few RR-Lyraes with near solar metallicities and disk-like kinematics exist. This is well in line with the models showing the deficiency in stars of the middle temperature range of the horizontal branch also in the data.

The bottom line is that our results actually fit to both scenarios, the binary evolution as well as the RGB-peel-off mechanism of D'Cruz et al. (1996). Therefore we cannot prove or disprove one or the other, even both could be in play. A still larger low z-sample, which does not suffer from selection effects against low $z_{\max }$ stars (see Sect. 2.1.1) could help answering this question. If the binary scenario is the dominating process leading to the forming of sdB stars, then the ratio of sdB stars belonging to the Halo, Thick and Thin Disk should be similar to that of other evolved stars. If, however, the D'Cruz et al. (1996) scheme holds true, then sdB stars in the Disk should be a little more numerous than expected. A really large and complete sample is required to find such subtle differences.

\section{Conclusions and outlook}

In this study we demonstrated that there are not only sdB stars belonging to the Disk, but that also a Halo Population exists. The scale height of $0.9 \mathrm{kpc}$ we derived for the Thick Disk is close to the middle of the range of values found in the literature. The trend in kinematics of stars along the horizontal branch described earlier (Altmann \& de Boer 2000) is still obvious in spite of the fact that we found a number of Halo sdB stars in this current study. This hints to somewhat different routes in development from the RGB to the HB for stars which become HBA and stars become sdB.

We have shown that $\mathrm{sdB}$ stars are a good tracer for the older populations. They are relatively numerous in the Thick Disk and present in the Halo. Some more problematic issues remain. Due to the binary nature (with the secondary component unseen) of a significant fraction of sdB stars, individual objects may have a measured radial velocity which is far off the actual systemic value. Another point is that we only had one first epoch position at our disposal. Therefore systemic radial velocities and new (possibly satellite based) proper motions should be obtained in the future. Apart from this, sdB stars at low Galactic latitudes should be included in a similar study, to investigate the Thin Disk population. Many more (even close by) sdB stars are not included, mostly because no measured radial velocity is available (although some of these have Tycho 2 or even Hipparcos proper motions). Analyses similar to this one could also be carried out for other HB-like objects, whose kinematics have not yet been studied in great detail. Studies concerning the kinetic behaviour of white dwarfs are underway (see, e.g. Pauli et al. 2003). Once satellite missions such as GAIA are completed, we will have a much larger sample with much more accurate data. This would enable us to confine principal morphological parameters very closely, and give us access to substructure in all populations discussed.

Acknowledgements. Part of this work was supported by the German Deutsche Forschungsgemeinschaft, DFG (Bo 779/21 and
He 1354/30-1). MA is supported by DLR grant No. 50QD 0102. We thank Tom Marsh (Southampton) for kindly supplying us with his new systemic radial velocities. MA whishes to thank those contributing to the data collection in this long range project, namely Oliver Cordes, Andrea Dieball, Jörg Sanner, Ralf Vanscheidt, Yolanda Aguilar-Sanchez, Ralf Kohley, Armin Theissen, Sabine Moehler, Michael Lemke and Patrick Francois, and the assistants supporting observations at Calar Alto and La Silla observatories. Furthermore we wish to thank Michael Geffert, Uli Heber, Ralf Napiwotzki and Michael Odenkirchen for many fruitful discussions, the kinematic and astrometric software or spectral models they readily supplied to us. With pleasure we made extensive use of the SIMBAD archive at CDS.

\section{References}

Allen, C., \& Santillan, A. 1991, RMxAA, 22, 255

Altmann, M., \& de Boer, K. S. 2000, A\&A, 353, 135

Aznar Cuadrado, R., \& Jeffery, C. S. 2002, A\&A, 385, 131

Beers, T. C., Doinidis, S. P., Griffin, K. E., Preston, G. W., \& Shectman, S. A. 1992, AJ, 103, 267

Behr, B. B., Cohen, J. G., McCarthy, J. K., \& Djorgovski, S. G. 1999, ApJ, 517, L135

Bergeron, P., Saffer, R. A., \& Liebert, J. 1992, ApJ, 394, 228

Bertin, E., \& Arnouts, S. 1996, A\&A, 117, 393

Bonifacio, P., Castelli, F., \& Hack, M. 1995, A\&AS, 110, 441

Brosche, P., Wildermann, E., \& Geffert, M. 1989, A\&A, 211, 239

Burstein, D., \& Heiles, C. 1982, AJ, 87, 1165

Carney, B. W., Laird, J. B., Latham, D. W., \& Aguilar, L. A. 1996, AJ, 112,668

Chen, B., Stoughton, C., Smith, J. A., et al. 2001, ApJ, 553, 184

Colin, J., de Boer, K. S., Dauphole, B., et al. 1994, A\&A, 287, 38

D'Cruz, N. L., Dorman, B., Rood, R. T., \& O'Connell, R. W. 1996, ApJ, 466, 359

de Boer, K. S., Aguilar Sanchez, Y., Altmann, M., et al. 1997, A\&A, 327, 577

Dinescu, D. I., Girard, T. M., \& van Altena, W. F. 1999a, AJ, 117, 1792

Dinescu, D. I., Girard, T. M., van Altena, W. F., Mendez, R. A., \& Lopez, C. E. 1997, AJ, 114, 1014

Dinescu, D. I., van Altena, W. F., Girard, T. M., \& López, C. E. 1999b, AJ, 117, 277

Dorman, B., Rood, R. T., \& O'Connell, R. W. 1993, ApJ, 419, 596

Edelmann, H., Christlieb, N., \& Heber, U. 2003, A\&A, in prep.

ESA. 1997, The Tycho and Hipparcos catalogue, vol. SP-1200 (ESA)

Geffert, M. 1998, A\&A, 340, 305

Geffert, M., Klemola, A. R., Hiesgen, M., \& Schmoll, J. 1997, A\&AS, 124,157

Green, R. F., Schmidt, M., \& Liebert, J. 1986, ApJS, 61, 305

Harris, W. E. 1996, AJ, 112, 1487

Heber, U., Reid, I. N., \& Werner, K. 2000, A\&A, 363, 198

Helmi, A., \& White, S. D. M. 1999, MNRAS, 307, 495

Høg, E., Fabricius, C., Makarov, V. V., et al. 2000, A\&A, 355, L27

Iben, I. J., \& Tutukov, A. V. 1987, ApJ, 313, 727

Irwin, M., Maddox, S., \& McMahon, R. 1994, Spectrum, 2, 14

Irwin, M., \& McMahon, R. 1992, Gemini, 37, 1

Kerber, L. O., Javiel, S. C., \& Santiago, B. X. 2001, A\&A, 365, 424

Kilkenny, D., Heber, U., \& Drilling, J. S. 1988, South African Astronomical Observatory Circular, 12, 1

Koen, C., O’Donoghue, D., Kilkenny, D., Stobie, R. S., \& Saffer, R. A. 1999, MNRAS, 306, 213

Lamontagne, R., Demers, S., Wesemael, F., Fontaine, G., \& Irwin, M. J. 2000, AJ, 119, 241 
Landolt, A. 1992, AJ, 104, 340

Lemke, M. 1997, A\&AS, 122, 285

Maxted, P. F. L., Heber, U., Marsh, T. R., \& North, R. C. 2001, MNRAS, 326, 1391

Moehler, S., Richtler, T., de Boer, K. S., Dettmar, R. J., \& Heber, U. 1990, A\&AS, 86, 53

Moehler, S., Sweigart, A. V., Landsman, W. B., Heber, U., \& Catelan, M. 1999, A\&A, 346, L1

Monet, D. E. A. 1998, in The PMM USNO-A2.0 Catalog. (1998), 0

Morales-Rueda, L., Maxted, P., Marsh, T., North, R., \& Heber, U. 2003, MNRAS, 338, 752

Moran, C., Maxted, P., Marsh, T. R., Saffer, R. A., \& Livio, M. 1999, MNRAS, 304, 535

Napiwotzki, R. 1997, A\&A, 322, 256

Ojha, D. 1994, in The Formation of the Milky Way Proc. of the IAAIAC-University of Pisa Workshop, Granada, Spain, 4.-9.9.1994, ed. E. J. Alfaro, \& A. J. Delgado (Granada, Spain: Instituto de Astrofísica de Andalucía), 215

Ojha, D. K., Bienayme, O., Robin, A. C., \& Mohan, V. 1994, A\&A, 284,810

Oke, J. B. 1990, AJ, 99, 1621
Pauli, E.-M., Napiwotzki, R., Altmann, M., et al. 2003, A\&A, 400, 877

Phleps, S., Meisenheimer, K., Fuchs, B., \& Wolf, C. 2000, A\&A, 356, 108

Reid, N., \& Majewski, S. R. 1993, ApJ, 409, 635

Reylé, C., \& Robin, A. C. 2001, A\&A, 373, 886

Saffer, R. A., Bergeron, P., Koester, D., \& Liebert, J. 1994, ApJ, 432, 351

Schlegel, D. J., Finkbeiner, D. P., \& Davis, M. 1998, ApJ, 500, 525

Schuster, W. J., \& Allen, C. 1997, A\&A, 319, 796

Slettebak, A., \& Brundage, R. K. 1971, AJ, 76, 338

Theissen, A., Moehler, S., Heber, U., Schmidt, J. H. K., \& de Boer, K. S. 1995, A\&A, 298, 577

Thejll, P., Flynn, C., Williamson, R., \& Saffer, R. 1997, A\&A, 317, 689

Thejll, P., Theissen, A., \& Jimenez, R. 1994, A\&A, 292, 457

Tüg, H. 1977, The Messenger, 11, 7

Unglaub, K., \& Bues, I. 1990, A\&A, 233, 159

Vivas, A. K., Zinn, R., Andrews, P., et al. 2001, ApJ, 554, L33

Yanny, B., Newberg, H. J., Kent, S., et al. 2000, ApJ, 540, 825 